\title{
Modeling and Analysis of Networked Control Systems using Stochastic Hybrid Systems*
}

\author{
João P. Hespanha ${ }^{\dagger}$
}

September 3, 2014

\begin{abstract}
This paper aims at familiarizing the reader with Stochastic Hybrid Systems (SHSs) and enabling her to use these systems to model and analyze Networked Control Systems (NCSs). Towards this goal, we introduce two different models of SHSs and a set of theoretical tools for their analysis. In parallel with the presentation of the mathematical models and results, we provide a few simple examples that illustrate the use of SHSs to models NCSs.
\end{abstract}

Keywords: Network Control Systems; Hybrid Systems; Stochastic Processes; Stability; Markov Processes

\section{Networked Control Systems}

The expression Networked Control Systems (NCSs) typically refers to feedback control systems for which some of the sensors, controllers, and actuators communicate with each other using a shared communication network. The use of a multi-purpose shared network reduces installation and maintenance costs and adds flexibility, as it permits the system reconfiguration and/or expansion with minimal additional infrastructure costs. In view of this, NCSs are finding application in numerous areas that include the automotive industry, the aviation industry, robotics, process control, and building control, among others.

While NCSs are attractive from the perspective of cost of deployment and maintenance, they introduce significant design challenges, because the traditional unity feedback loop that operates in continuous time or at a fixed sampling rate is not adequate when sensor data arrives from multiple sources, asynchronously, delayed, and possibly corrupted. Consequently, NCSs have been the focus of intense study in the last few years [16, 25, 29].

This paper is focused on two aspects of NCSs that are responsible for important challenges in analyzing and designing NCSs and that are prompting the development of new formal tools to study these systems.

\footnotetext{
* This paper is partially based on material presented in a plenary lecture at the 4th IFAC Workshop on Distributed Estimation and Control in Networked Systems (NECSYS'13).

${ }^{\dagger}$ This material is based upon work supported by the National Science Foundation under Grants No. EF-1137835 and CNS-1329650, and by the Institute for Collaborative Biotechnologies through grant W911NF-09-0001 from the U.S. Army Research Office.
} 


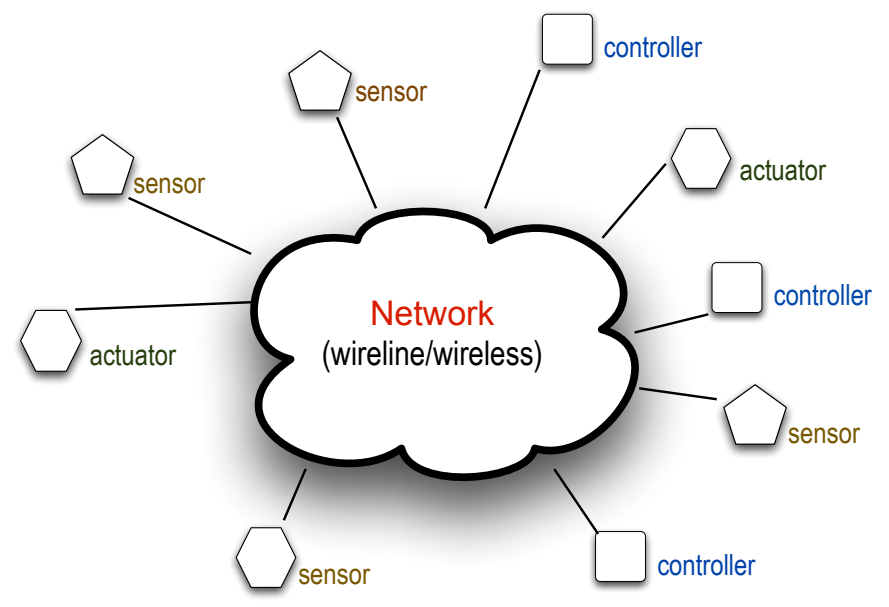

Figure 1: Networked Control System (NCS)

1. In NCSs, the information that flows between different elements in the feedback loop (sensors, actuators, or controllers) is carried in atomic units called packets; and the departure and arrival of these packets causes instantaneous changes in the state of the system. Moreover, the protocols used to process packets often contains discrete or logic variables, which must be taken into account in their analysis.

2. The shared network that supports the communication between the different elements of a NCS introduces uncertainty that typically arises from other (unmodeled) systems that compete for network resources as well as from stochasticity introduced explicitly by the network protocols (e.g., random backoffs in response to packet collisions).

The integration of continuous dynamics with discrete variables (often boolean) requires the use of "hybrid" models for dynamical systems, whose solutions should include "stochastic behavior" to capture the uncertainty common in NCSs. These requirements motivate the use of Stochastic Hybrid Systems (SHSs) as a natural modeling formalism for NCSs.

The goal of this tutorial paper is twofold: First, we aim at familiarizing the reader with SHSs as a modeling framework for NCSs. Second, we want to introduce a few theoretical tools that we found instrumental for the analysis and design of NCSs feedback controllers and network protocols. We shall attempt to make the presentation tutorial but rigorous, and incorporate in it a few examples to illustrate the use of the models and tools.

Towards the above mentioned goals, Section 2 introduces the concept of SHSs. We start with a deterministic hybrid model, whose state has a component that evolves continuously and another that is discrete in nature, changing through instantaneous resets or impulses (Section 2.1). The key mechanism used here to introduce stochasticity is through the times at which impulses take places, which in the context of networked control systems, often correspond to the times at which messages are sent or received from the network. In the first (and simpler) model considered, denoted by Timetriggered Stochastic Hybrid Systems (TTSHSs), the reset times may causally depend on the hybrid system's discrete state, but are not allowed to depend on its continuous state (Section 2.2). This 
restriction is removed in a second model for stochastic hybrid systems, denoted by State-Driven Stochastic Hybrid Systems (SDSHSs), where a (causal) dependence on the full state is allowed (Section 2.3). For both models, we provide algorithms to construct the sample paths for the SHSs. These algorithms provide the basis for the formal analysis of these systems and can also be used to generate Monte Carlo simulations for the SHSs.

In parallel with the introduction of formal models for SHSs, we provide two examples that illustrate the use of SHSs to model networked control systems. One example consists of a feedback loop with multiple sensors that communicate with a controller through a shared network (Example 1) and the other refers to the estimation of the state of a system based on measurements received through a packet-switched network (Example 2).

The formal tools presented here to analyze SHSs are focused on establishing global existence of solution, stability, and on constructing bounds on the state of the system. There is a wide range of stability notions applicable to SHSs [23] and we review some of the most commonly used ones in Section 3. The stability notions considered can be classified in two broad categories: bounds on the probability that the state of the system "misbehaves" or bounds on the expected value of the state.

The stability theory for linear TTSHSs is fairly well established and simple necessary and sufficient stability conditions are available. These conditions are presented in Section 4 for the case of a single discrete state, but they have been generalized to multiple discrete states [3].

The key technical tools discussed in Section 5 for the formal analysis of nonlinear TTSHS and SDSHS are based on the concepts of extended generator and Lyapunov function. Essentially, the extended generator of a SHS provides a formula (known as Dynkin's formula) that can be used to compute the instantaneous value of the derivative of the expected value of a function $V$ that dependes on the (continuous and discrete) state of the SHS; much like the chain rule allows one to compute the time derivative of a function $V(x)$ along solutions to a (deterministic) ODE of the form $\dot{x}=f(x)$ (Section 5.1). Section 5.3 provides several Lyapunov-based conditions that can be used to establish global existence, stability, and boundedness for SHSs. These conditions are expressed in terms of algebraic inequalities on "candidate Lyapunov functions" that greatly resemble the conditions that appear in Lyapunov's stability theorem for deterministic ODEs.

While the extended generator and Dynkin's formula are powerful tools for the analysis of SHSs, their direct applicability requires stringent assumptions, which often cannot be established independently. A solution to this technical difficult relies in considering a "stopped" process that matches the solution to the SHS until the first time it leaves a bounded set and gets "frozen" at the precise time it leaves the set. Two key features make stopped processes especially useful: (i) Dynkin's formula holds for stopped processes under very mild assumptions and (ii) the stopped process converges to the state of the hybrid system as one enlarges the set outside which the process gets frozen. The use of stopped processes to study SHSs is discussed in Section 5.2. While the main goal of this paper is not to provide the full formal proofs behind the tools prevented, we include in Section 5.4 the key steps behind the Lyapunov-based proofs. Our goal is to provide the reader with the basic tools needed to construct variations of the results presented here, instead of attempting to provide a long (and somewhat boring) list of all possible variations. However, the stability results presented in Section 5.3 are self-contained in that using these results does not require the discussion in Sections 5.2 and 5.4. 
The present paper aims at being a tutorial on SHSs and not an exhaustive literature survey on SHSs. For the latter, we refer the reader to the excellent recent survey [23].

Notation All random variables in this write-up are measurable on the same probability space $(\Omega, \mathcal{F}, \mathrm{P})$. We denote random variables and stochastic processes in boldface and, for short of notation, we generally omit the dependence on the outcome $\omega \in \Omega$ [as in $\mathbf{t}_{k}$ or $\mathbf{N}(t)$, instead of $\mathbf{t}_{k}(\omega)$ or $\mathbf{N}(t ; \omega)$ ]. In expressions involving random variable that require some quantification on

$\omega$, we use the superscript ${ }^{\text {wpo }}$ to denote universal quantification with respect to some subset of $\Omega$ with probability one [as in $\mathbf{X} \stackrel{\text { wpo }}{=} \mathbf{Z}$ to express that $\exists \bar{\Omega} \in \mathcal{F}$ such that $\mathrm{P}(\bar{\Omega})=1$ and $\mathbf{X}(\omega)=\mathbf{Y}(\omega)$, $\forall \omega \in \bar{\Omega}$.] A time-dependent function $x:[0, T) \rightarrow \mathbb{R}^{n}, T \in(0, \infty]$ that is continuous from the right and has well-defined limits from the left for every time $t \in[0, T)$ is said to be cadlag (from French "continue à droite, limite à gauche"). For cadlag functions, we denote by $(\cdot)^{-}$the left-limit operator that maps $x$ into the function $x^{-}:(0, T) \rightarrow \mathbb{R}^{n}$ defined by $x^{-}(t):=\lim _{\tau \downarrow t} x(\tau), \forall t \in(0, T)$.

\section{Stochastic Hybrid Systems}

Hybrid Systems (HSs) combine continuous-time dynamics with discrete modes of operation. The states of such systems usually have two distinct components: one that evolves continuously, typically according to a differential equation; and another one that is discrete and changes through instantaneous resets or impulses. The Stochastic Hybrid Systems (SHSs) considered here can be viewed as HSs for which the resets are triggered by stochastic events, much like transitions between states of a continuous-time Markov chains. However, the rate at which resets occur is allowed to depend on both the continuous and the discrete states of the SHS.

SHSs have been introduced in [17] and further developed in [6, 7, 14, 15, 22]. Here, we consider a restricted form of SHSs that are closely related to (and heavily inspired by) the PiecewiseDeterministic Markov Process (PDMPs) introduced by Davis [11] and, in fact, our SHSs can be viewed as a special case of PDMPs and thus inherent many of the PDMPs properties. SHSs are also closely related to Markov Jump Linear Systems (MJLS) [10, 20] and to Switching Difusions (SD) $[13,27]$, which differ from our SHSs in that the emphasis in MJLSs and SDs is in the change in dynamics at a set of event times and not on the impulsive effects that are fundamental in many NCSs. Nevertheless, MJLSs have been successfully used to study fairly complex NCSs [8]. Also, in SDs, the solution between discrete events is obtained from a stochastic differential equation, instead of an ordinary differential equation; therefore such systems exhibit randomness even without resets. The reader is referred [23] for a recent comprehensive survey on SHSs that discusses the key differences between these and related models that have appeared in the literature.

In the remainder of this section, we first introduce a simple model for deterministic hybrid systems and then explore a few mechanisms to introduce stochasticity into their solutions that are particularly useful in the context of NCSs.

\subsection{Deterministic Hybrid Systems}

We consider hybrid systems (HS) whose continuous state $x(t), t \geqslant 0$ takes values in $\mathbb{R}^{n}$ and whose discrete state $q(t), t \geqslant 0$ takes value in a (typically) discrete state $\mathcal{Q}$. When $q(t)$ takes some value $q_{1} \in \mathcal{Q}$, we say that the HS is in mode $q_{1}$ and when $q(t)$ changes from some $q_{1} \in \mathcal{Q}$ to another 
$q_{2} \in \mathcal{Q}$, we say that the HS transitioned from mode $q_{1}$ to mode $q_{2}$. A HS for which $\mathcal{Q}$ has a single element (and therefore the system is always in the same mode) is called an impulsive system (IS). In these hybrid systems, $q(t)$ is necessarily always equal to the only element of $\mathcal{Q}$ and therefore we generally omit any reference to their discrete state.

The simple model of deterministic hybrid systems (DHSs) considered here requires three ingredients:

1. a family of (locally Lipschitz) vector fields $\left\{f_{q}: \mathbb{R}^{n} \rightarrow \mathbb{R}^{n}, q \in \mathcal{Q}\right\}$ that defines the evolution of the continuous state $x(t)$ in each mode $q \in \mathcal{Q}$;

2. a family of reset maps $\left\{\phi_{\ell}: \mathcal{Q} \times \mathbb{R}^{n} \rightarrow \mathcal{Q} \times \mathbb{R}^{n}, \ell \in \mathcal{L}\right\}$ that characterizes how the resets or impulses change the system's state; and

3. a family of guards $\left\{g_{\ell}: \mathcal{Q} \times \mathbb{R}^{n} \rightarrow \mathbb{R}, \ell \in \mathcal{L}\right\}$ that defines when resets or impulses take place.

The families of reset maps and guards are parameterized by the same (typically finite) set $\mathcal{L}$ so that, for each $\ell \in \mathcal{L}$, the guard $g_{\ell}$ is associated with the reset map $\phi_{\ell}$.

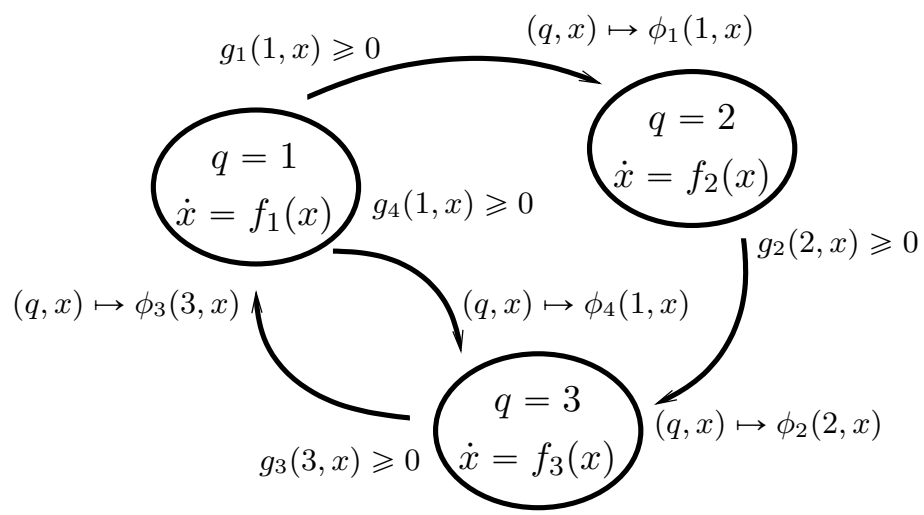

(a) (Multi-mode) Hybrid System (HS)

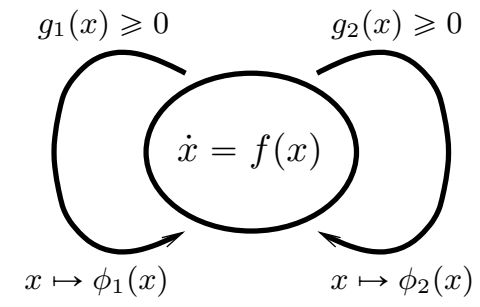

(b) Impulsive System (IS)

Figure 2: Deterministic Hybrid Systems (DHS)

Figure 2 shows a graphical representation of a DHS. Each elipse corresponds to a specific value of the discrete state in $\mathcal{Q}$ and is labelled with the differential equation for the continuous state $x(t)$ within the corresponding mode. The arrows are associated with transitions between modes and each arrow corresponds to an element of $\mathcal{L}$ (and therefore to a pair guard/reset map). The guard condition $g_{\ell}(q, x) \geqslant 0$ appearing at the start of an arrow indicates when the transition is triggered and the reset map $(q, x) \mapsto \phi_{\ell}(q, x)$ appearing at the end of the arrow specifies how the transition changes the system's state.

To construct a solution to this DHS, starting at an initial condition $q(0)=q_{0} \in \mathcal{Q}, x(0)=$ $x_{0} \in \mathbb{R}^{n}$, we solve the differential equation corresponding to the mode $q_{0}$ until one of the guard conditions becomes active (i.e., until one of the $g_{\ell}$ becomes positive or zero). At that time, we reset the state according to the corresponding reset map $\phi_{\ell}$ and proceed by solving the differential equation corresponding to the new mode. This procedure can be formalized as follows:

1. Set $k=0, t_{0}=0$. 
2. Solve the initial-value problem $z\left(t_{k}\right)=x\left(t_{k}\right), \dot{z}=f_{q\left(t_{k}\right)}(z), \forall t \geqslant t_{k}$; and let $\left[t_{k}, T\right)$ denote its maximum interval of existence.

3. If any one of the $g_{\ell}\left(q\left(t_{k}\right), z(t)\right)$ is non-negative at time $t_{k}$ or two or more of the $g_{\ell}\left(q\left(t_{k}\right), z(t)\right)$ became non-negative simultaneously in the interval $\left(t_{k}, T\right)$, then this procedure fails ${ }^{1}$.

4. If a single $g_{\ell}\left(q\left(t_{k}\right), z(t)\right), \ell \in \mathcal{L}$ becomes non-negative in the interval $\left(t_{k}, T\right)$, i.e.,

$$
t_{k+1}:=\inf \left\{t \in\left(t_{k}, T\right): \exists \ell \in \mathcal{L}, g_{\ell}\left(q\left(t_{k}\right), z(t)\right) \geqslant 0\right\}
$$

belongs to the (open) interval $\left(t_{k}, T\right)$ and $\exists \ell_{k} \in \mathcal{L}$ such that

$$
g_{\ell_{k}}\left(q\left(t_{k}\right), z\left(t_{k+1}\right)\right) \geqslant 0, \quad g_{\ell}\left(q\left(t_{k}\right), z\left(t_{k+1}\right)\right)<0, \quad \forall \ell \neq \ell_{k} ;
$$

then define the DHS state in the interval $\left[t_{k}, t_{k+1}\right)$ to be

$$
q(t)=q\left(t_{k}\right), x(t)=z(t), \quad \forall t \in\left[t_{k}, t_{k+1}\right)
$$

and at the time $t_{k+1}$ to be

$$
\left(q\left(t_{k+1}\right), x\left(t_{k+1}\right)\right)=\phi_{\ell_{k}}\left(q\left(t_{k}\right), z\left(t_{k+1}\right)\right) \quad\left(=\phi_{\ell_{k}}\left(q^{-}\left(t_{k+1}\right), x^{-}\left(t_{k+1}\right)\right)\right)
$$

Increment the integer $k$ and go back to 2 .

5. If none of the $g_{\ell}\left(q\left(t_{k}\right), z(t)\right), \ell \in \mathcal{L}$ becomes non-negative in the interval $\left[t_{k}, T\right)$, then define the DHS state in the interval $\left[t_{k}, T\right)$ to be

$$
q(t)=q\left(t_{k}\right), x(t)=z(t), \quad \forall t \in\left[t_{k}, T\right)
$$

and terminate.

The times $t_{k}$ are called reset times and this solution is defined up to a maximal time $T_{\max }$ that can be either equal to $T$ if the construction terminate through 5 or $\sup _{k} t_{k}$ if it does not terminate. In this and in all subsequent constructions of solutions to HSs the state variables $x(t)$ and $q(t)$ are typically not continuous at the reset times $t_{k}$, but they are continuous from the right and have well-defined limits from the left. Signals of this type are called cadlag (from French "continue à droite, limite à gauche").

Many models for DHSs have been proposed in the literature. The one described here is a simplification of previous models and, most notably, lacks non-determinism, i.e., the ability to allow solutions that may or may not reset when guards become active. The construction outlined above leads to uniqueness of solution, which somewhat simplifies the introduction of stochasticity. However, non-determinism can be combined with stochasticity as shown in [22].

\footnotetext{
${ }^{1}$ One can formulate conditions that are sufficient for the construction not to fail, but since the emphasis of this paper is in stochastic hybrid systems, we do not pursue that here.
} 


\subsection{Time-Triggered Stochastic Hybrid Systems}

In the DHS's considered in Section 2.1, the times $t_{k}$ at which the state is reset according to (1) correspond to the times at which one of the guards $g_{\ell}$ becomes non-negative. In Time-Triggered Stochastic Hybrid Systems (TTSHS), these $\mathbf{t}_{k}$ 's are random variables with the property that the intervals $\mathbf{h}_{k}:=\mathbf{t}_{k+1}-\mathbf{t}_{k}, \forall k \in \mathbb{Z}_{\geqslant 0}$ between consecutive resets are independent random variables with a probability distribution that may depend on the current mode. These hybrid systems are also known as Stochastic Hybrid Systems with Renewal Transitions [2, 3] because the stochastic process $\mathbf{N}(T)$ that counts the number of resets $\mathbf{t}_{k}$ in the interval $(0, T]$ is a renewal process.

The model of a TTSHS thus requires three ingredients:

1. a family of (locally Lipschitz) vector fields $\left\{f_{q}: \mathbb{R}^{n} \rightarrow \mathbb{R}^{n}, q \in \mathcal{Q}\right\}$ that defines the evolution of the continuous state $x(t)$ in each mode $q \in \mathcal{Q}$;

2. a family of reset maps $\left\{\phi_{\ell}: \mathcal{Q} \times \mathbb{R}^{n} \rightarrow \mathcal{Q} \times \mathbb{R}^{n}, \ell \in \mathcal{L}\right\}$ that characterizes how the resets or impulses change the system's state; and

3. a family of reset-time distributions $\left\{\mu_{q}^{\ell}: \ell \in \mathcal{L}, q \in \mathcal{Q}\right\}$ that defines when resets or impulses take place.

Also here, the families of reset maps and reset-time distributions are parameterized by the same (typically finite) set $\mathcal{L}$ so that, for each $\ell \in \mathcal{L}$, the reset-time distributions $\mu_{q}^{\ell}, q \in \mathcal{Q}$ are associated with the reset map $\phi_{\ell}$.

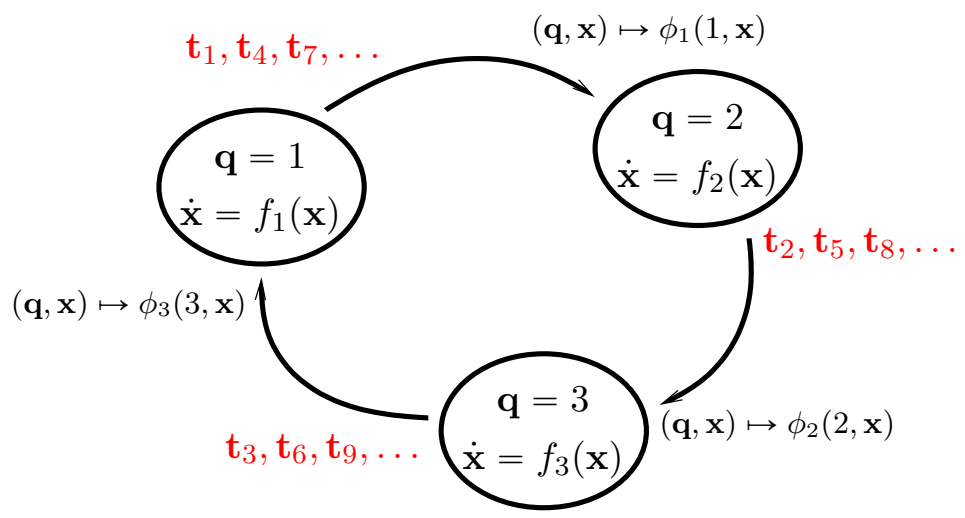

Figure 3: Time-Triggered Stochastic Hybrid System (TTSHS)

Figure 3 shows a graphical representation of a TTSHS: The key difference with respect to the DHS in Figure 2 is that now the guard conditions have been replaced by the random variables $\mathbf{t}_{k}$ that represent the reset times, which are determined by the reset-time distributions $\mu_{q}^{\ell}, \ell \in \mathcal{L}, q \in \mathcal{Q}$.

To construct a solution to this TTSHS, starting at an initial condition $\mathbf{q}(0)=q_{0} \in \mathcal{Q}, \mathbf{x}(0)=$ $x_{0} \in \mathbb{R}^{n}$, we draw one random number $\mathbf{h}_{0}^{\ell}, \ell \in \mathcal{L}$ for each reset-time distribution $\mu_{q(0)}^{\ell}, \ell \in \mathcal{L}$ and solve the differential equation corresponding to the mode $q_{0}$ for a time interval equal to the smallest of the $\mathbf{h}_{0}^{\ell}$. When that interval elapses, we reset the state according to the reset map $\phi_{\ell}$ corresponding to the smallest $\mathbf{h}_{0}^{\ell}$ and proceed by solving the differential equation corresponding to the new mode. Formally, the construction of the solution to a TTSHS is analogous to the 
construction described in Section 2.1 for a DHS, but with the new (stochastic) mechanism used to generate the reset times. Specifically, the steps $2-5$ should now be replaced by:

2'. Solve the initial-value problem $\mathbf{z}\left(\mathbf{t}_{k}\right)=\mathbf{x}\left(\mathbf{t}_{k}\right), \dot{\mathbf{z}}=f_{\mathbf{q}\left(t_{k}\right)}(\mathbf{z}), \forall t \geqslant \mathbf{t}_{k}$; and let $\left[\mathbf{t}_{k}, \mathbf{T}\right)$ denote its maximum interval of existence.

3'. Draw independent random numbers $\mathbf{h}_{k}^{\ell}, \ell \in \mathcal{L}$ with distributions $\mu_{\mathbf{q}\left(\mathbf{t}_{k}\right)}^{\ell}$. If any of the $\mathbf{h}_{k}^{\ell}$ is equal to zero or more than one of the $\mathbf{h}_{k}^{\ell}$ is equal to $\mathbf{h}_{k}:=\min _{\ell \in \mathcal{L}} \mathbf{h}_{k}^{\ell}$ then this procedure fails. Otherwise set

$$
\mathbf{t}_{k+1}:=\mathbf{t}_{k}+\mathbf{h}_{k} .
$$

4'. If $\mathbf{t}_{k+1} \in\left(\mathbf{t}_{k}, \mathbf{T}\right)$ and a single $\mathbf{h}_{k}^{\ell}$ is equal to $\mathbf{h}_{k}$, i.e., $\exists \boldsymbol{\ell}_{k} \in \mathcal{L}$ such that

$$
\mathbf{h}_{k}^{\ell_{k}}=\mathbf{h}_{k}, \quad \mathbf{h}_{k}^{\ell}>\mathbf{h}_{k}, \quad \forall \ell \neq \ell_{k} ;
$$

then define the TTSHS state in the interval $\left[\mathbf{t}_{k}, \mathbf{t}_{k+1}\right)$ to be

$$
\mathbf{q}(t)=\mathbf{q}\left(\mathbf{t}_{k}\right), \mathbf{x}(t)=\mathbf{z}(t), \quad \forall t \in\left[\mathbf{t}_{k}, \mathbf{t}_{k+1}\right)
$$

and at the time $\mathbf{t}_{k+1}$ to be

$$
\left(\mathbf{q}\left(\mathbf{t}_{k+1}\right), \mathbf{x}\left(\mathbf{t}_{k+1}\right)\right)=\phi_{\boldsymbol{\ell}_{k}}\left(\mathbf{q}\left(\mathbf{t}_{k}\right), \mathbf{z}\left(\mathbf{t}_{k+1}\right)\right) \quad\left(=\phi_{\boldsymbol{\ell}_{k}}\left(\mathbf{q}^{-}\left(\mathbf{t}_{k+1}\right), \mathbf{x}^{-}\left(\mathbf{t}_{k+1}\right)\right)\right)
$$

Increment the integer $k$ and go back to 2 '.

5'. If $\mathbf{t}_{k+1} \geqslant \mathbf{T}$, then define the TTSHS state in the interval $\left[\mathbf{t}_{k}, \mathbf{T}\right)$ to be

$$
\mathbf{q}(t)=\mathbf{q}\left(\mathbf{t}_{k}\right), \mathbf{x}(t)=\mathbf{z}(t), \quad \forall t \in\left[\mathbf{t}_{k}, \mathbf{T}\right)
$$

and terminate.

Also here, the random times $\mathbf{t}_{k}$ are called reset times and this solution is defined up to a maximal time $\mathbf{T}_{\max }$ that can be either equal to $\mathbf{T}$ if the construction terminates through ${ }^{\prime}$ ' or $\sup _{k} \mathbf{t}_{k}$ if it does not terminate.

The following assumptions can be used to make sure that this construction does not fail in step 3 ' with probability 1 ; either because we get an $\mathbf{h}_{k}^{\ell}$ equal to zero or because more than one of the $\mathbf{h}_{k}^{\ell}$ is equal to $\mathbf{h}_{k}:=\min _{\ell \in \mathcal{L}} \mathbf{h}_{k}^{\ell}$.

Assumption 1 (TTSHS Standing Assumptions). The following is assumed for the TTSHSs considered throughout this paper:

A1 The vector fields $x \mapsto f_{q}(x), q \in \mathcal{Q}$ are locally Lipschitz.

A2 $\mathrm{P}\left(\mathbf{h}_{k}^{\ell}=0\right)=0$ for every $\ell \in \mathcal{L}, k \in \mathbb{Z}_{\geqslant 0}$, i.e., none of the reset-time distributions $\mu_{q}^{\ell}, \ell \in \mathcal{L}$, $q \in \mathcal{Q}$ have a point mass at 0 .

A3 $\mathrm{P}\left(\mathbf{h}_{k}^{\ell_{1}}=\mathbf{h}_{k}^{\ell_{2}}\right)=0$ for every $\ell_{1} \neq \ell_{2}, k \in \mathbb{Z}_{\geqslant 0}$, i.e., for every $q \in \mathcal{Q}$ and $\ell_{1} \neq \ell_{2}$ the reset-time distributions $\mu_{q}^{\ell_{1}}, \mu_{q}^{\ell_{2}}$ cannot have common point masses. 


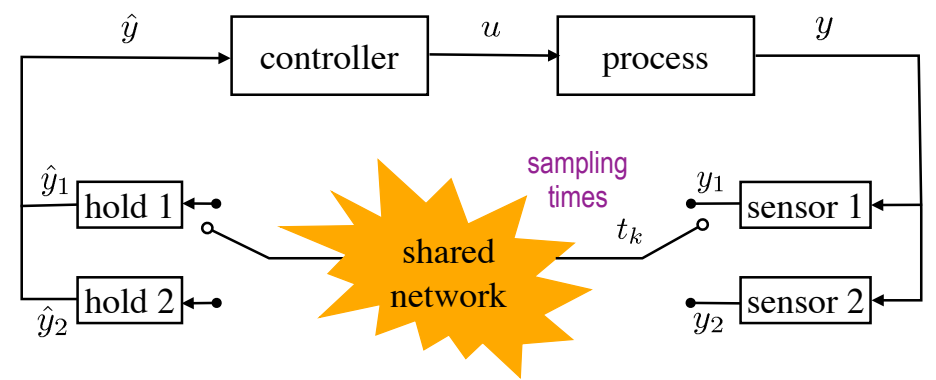

Figure 4: Feedback control loop with two remote sensors that communicate with the controller through a shared network in Example 1.

Example 1 (Estimation through a packet-switched network). Figure 4 depicts a networked control system that could be modeled using a TTSHS. It corresponds to a feedback configuration in which the process output vector $y$ can be decomposed into two components $y_{1}$ and $y_{2}$, each measured by a separate sensor. The two sensors are located far from the controller and communicate with it through a shared network.

In this example, we assume that the sensors take turns in sampling the output and sending their measurements to the controller. Specifically, the process output $y$ is sampled at times $\mathbf{t}_{k}$, $k \in \mathbb{Z}_{\geqslant 0}$ and sensor 1 sends the value of $y_{1}$ at times $\mathbf{t}_{k}$ with $k$ odd whereas sensor 2 sends the value of $y_{2}$ at times $\mathbf{t}_{k}$ with $k$ even. Between sampling times, the controller simply holds the values of the measurements received. This leads to a discrepancy between the value of the process output $y(t)$ and the input to the controller $\hat{y}(t)$, at each time $t$. Assuming linear time-invariant (LTI) models for the process and the controller of the form:

$$
\begin{aligned}
\dot{x}_{P} & =A_{P} x_{P}+B_{P} u, & \dot{x}_{C} & =A_{C} x_{C}+\left[\begin{array}{ll}
B_{1} & B_{2}
\end{array}\right]\left[\begin{array}{l}
\hat{y}_{1} \\
\hat{y}_{2}
\end{array}\right] \\
{\left[\begin{array}{l}
y_{1} \\
y_{2}
\end{array}\right] } & =\left[\begin{array}{l}
C_{1} \\
C_{2}
\end{array}\right] x_{P}, & u & =C_{C} x_{C},
\end{aligned}
$$

the system dynamics can be written as

$$
\dot{x}=A x, \quad \text { where } \quad x:=\left[\begin{array}{c}
x_{P} \\
x_{C} \\
\hat{y}_{1} \\
\hat{y}_{2}
\end{array}\right], \quad A:=\left[\begin{array}{cccc}
A_{P} & B_{P} C_{c} & 0 & 0 \\
0 & A_{C} & B_{1} & B_{2} \\
0 & 0 & 0 & 0 \\
0 & 0 & 0 & 0
\end{array}\right],
$$

in any interval $\left[\mathbf{t}_{k}, \mathbf{t}_{k+1}\right), k \in \mathbb{Z}_{\geqslant 0}$ between sampling times; and at each sampling time $\mathbf{t}_{k} \in \mathbb{Z}_{\geqslant 1}$, we have

$$
x\left(\mathbf{t}_{k}\right):=\left\{\begin{array}{ll}
J_{\text {odd }} x^{-}\left(\mathbf{t}_{k}\right) & k \text { odd } \\
J_{\text {even }} x^{-}\left(\mathbf{t}_{k}\right) & k \text { even, }
\end{array} \quad J_{\text {odd }}:=\left[\begin{array}{cccc}
I & 0 & 0 & 0 \\
0 & I & 0 & 0 \\
C_{1} & 0 & 0 & 0 \\
0 & 0 & 0 & I
\end{array}\right], \quad J_{\text {even }}:=\left[\begin{array}{cccc}
I & 0 & 0 & 0 \\
0 & I & 0 & 0 \\
0 & 0 & I & 0 \\
C_{2} & 0 & 0 & 0
\end{array}\right]\right.
$$


which expresses the fact that $x_{P}$ and $x_{C}$ are continuous signals and, at odd sampling times, $\hat{y}_{1}\left(\mathbf{t}_{k}\right)$ is updated to the value of $y_{1}\left(\mathbf{t}_{k}\right)=C_{1} x_{P}\left(\mathbf{t}_{k}\right)$, whereas $\hat{y}_{2}\left(\mathbf{t}_{k}\right)$ remains with the same value. The roles are reversed at the even sampling times.

Typically, the sensors would take measurements in a periodic fashion, e.g., with $\mathbf{t}_{k+1}-\mathbf{t}_{k}$ always equal to some (deterministic) constant $T_{s}$. However, when data is sent through a shared network, the sensors are unable to send data if the network is busy and may need to wait until data can be transmitted. Assuming that, when the shared network becomes available at time $\mathbf{t}_{k}$, the appropriate sensor sends the current output $y_{1}\left(\mathbf{t}_{k}\right)$ or $y_{2}\left(\mathbf{t}_{k}\right)$ (depending on wether $k$ is odd or even, respectively), the interval $\mathbf{h}_{k}:=\mathbf{t}_{k+1}-\mathbf{t}_{k}$ becomes a random variable due to the stochastic nature of the external network traffic and the network protocols. Note that in the case considered here, one would expect the distribution of $\mathbf{t}_{k}$ to have support in the interval $\left[T_{s}, \infty\right)$ and a point mass at $T_{s}$ with weight equal to the probability that the network is available at some fixed point in time.

This networked control system can be naturally modeled using the TTSHS in Figure 5. The system has two discrete states (modes) that keep track of which sensor should send its data. Both modes have the same vector field (5), but different reset maps defined by the matrices $J_{\text {odd }}$ and $J_{\text {even }}$ in (6). The reset maps

$$
\phi_{1}(q, x)=\left(2, J_{\text {odd }} x\right), \quad \phi_{2}(q, x)=\left(1, J_{\text {even }} x\right),
$$

are associated with the reset-time distributions $\mu_{1}^{1}(\cdot)$ and $\mu_{2}^{2}(\cdot)$ of the intervals $\mathbf{h}_{k}:=\mathbf{t}_{k+1}-\mathbf{t}_{k}$ for $k$ odd and even, respectively. This model implicitly assumes that consecutive $\mathbf{h}_{k}$ are statistically

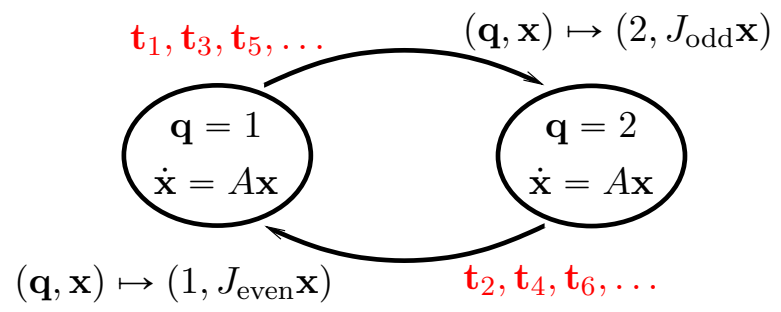

Figure 5: Time-Triggered Stochastic Hybrid System (TTSHS) that models the networked control system in Example 1.

independent, which means that the delay experienced by one sensor before sending the measurement at time $\mathbf{t}_{k}$ is independent of the delay experienced by the other sensor before sending the measurement at time $\mathbf{t}_{k+1}$. This may not be very realistic since a large delay could mean that the network is in a heavy-load condition and so subsequent delays would likely be high. However, this modeling problem could be avoided by considering more discrete modes. E.g., one could imagine that the network has 2 (or more) "traffic modes" and switches stochastically between these modes: one mode could correspond to low traffic and another to high traffic. This would lead to a TTSHS with 4 discrete states: $k$ even or odd and, for each option, the two network states. Packet drops could also be considered using an approach similar to that used in [2, Section V].

\subsection{State-Driven Stochastic Hybrid Systems}

In the TTSHS model described in Section 2.2, the reset times $\mathbf{t}_{k}$ are random variables whose distributions do not depend on the continuous state $\mathbf{x}(t)$. This is not to say that the $\mathbf{t}_{k}$ and $\mathbf{x}(t)$ 
are statistically independent, in fact, they typically are not. However, while the reset time $\mathbf{t}_{k}$ affects the construction of $\mathbf{x}(t), t \geqslant \mathbf{t}_{k}$; the distribution of the next reset time $\mathbf{t}_{k}>t$ is allowed to depend on the current discrete state $\mathbf{q}(t)$, but not on the current continuous state $\mathbf{x}(t)$. In State-Driven Stochastic Hybrid Systems (SDSHS) this is not the case and the continuous state $\mathbf{x}(t)$ of a SDSHS can (causally) affect the reset times by modulating the "instantaneous" probability of resets.

The model of a SDSHS also requires three ingredients:

1. a family of (locally Lipschitz) vector fields $\left\{f_{q}: \mathbb{R}^{n} \rightarrow \mathbb{R}^{n}, q \in \mathcal{Q}\right\}$ that defines the evolution of the continuous state $x(t)$ in each mode $q \in \mathcal{Q}$;

2. a family of reset maps $\left\{\phi_{\ell}: \mathcal{Q} \times \mathbb{R}^{n} \rightarrow \mathcal{Q} \times \mathbb{R}^{n}, \ell \in \mathcal{L}\right\}$ that characterizes how the jumps or impulses change the system's state; and

3. a family of transition intensities $\left\{\lambda_{\ell}: \mathcal{Q} \times \mathbb{R}^{n} \rightarrow[0, \infty), \ell \in \mathcal{L}\right\}$ that defines when jumps or impulses take place.

Also here, the families of reset maps and transition intensities are parameterized by the same (typically finite) set $\mathcal{L}$ so that, for each $\ell \in \mathcal{L}$, the transition intensity $\lambda_{\ell}$ is associated with the reset map $\phi_{\ell}$. Figure 6 shows a graphical representation of a SDSHS: The key difference with respect to

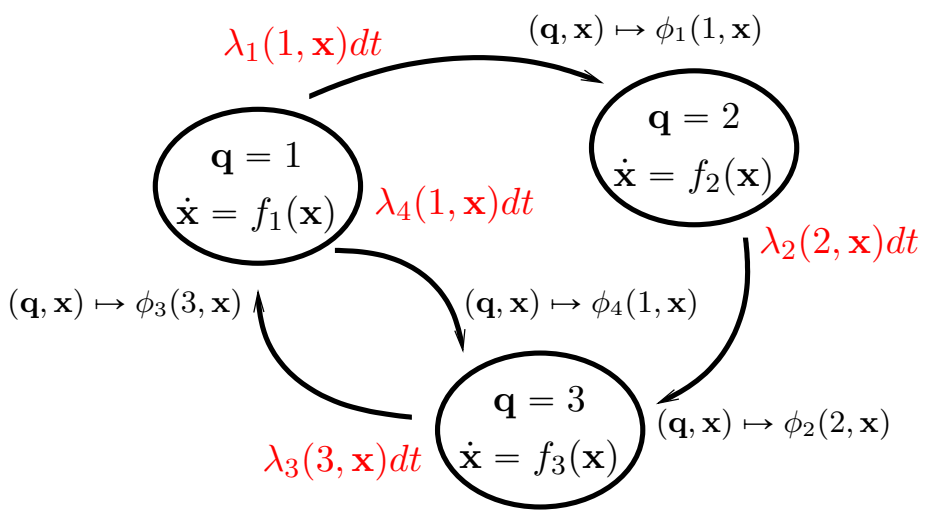

Figure 6: State-Driven Stochastic Hybrid System (TTSHS)

the HS in Figure 2 is that now the guard conditions have been replaced by the transition intensities $\lambda_{\ell}$ that determine the "instantaneous" probability of a reset. Specifically, given the SDSHS's state $\mathbf{q}(t), \mathbf{x}(t)$ at a time $t \geqslant 0$, the probability that the state will be reset by $\phi_{\ell}$ during the "elementary" interval $(t, t+d t]$ is given by $\lambda_{\ell}(\mathbf{q}(t), \mathbf{x}(t)) d t$. More specifically,

$$
\mathrm{P}\left(m \text { resets by } \phi_{\ell} \text { in }(t, t+d t] \mid \mathbf{q}(t), \mathbf{x}(t)\right)=\left\{\begin{array}{ll}
1-\lambda_{\ell}(\mathbf{q}(t), \mathbf{x}(t)) d t & m=0, \\
\lambda_{\ell}(\mathbf{q}(t), \mathbf{x}(t)) d t & m=1, \\
0 & m>1,
\end{array} \quad\left(\text { as } d t \downarrow 0^{+}\right) .\right.
$$

By an "elementary" time interval, we mean that these equalities hold as the length of the interval $d t$ converges to zero. One can also think of $\lambda_{\ell}(\mathbf{q}(t), \mathbf{x}(t))$ as the "instantaneous" average number of resets by $\phi_{\ell}$ per unit of time.

The construction of a solution to this SDSHS, starting at an initial condition $q(0)=q_{0} \in \mathcal{Q}$, $x(0)=x_{0} \in \mathbb{R}^{n}$, is analogous to the one described in Section 2.1 for a DHS, but with a new 
stochastic mechanism used to generate the reset times. Specifically, the steps $2-5$ should now be replaced by:

2". Solve the initial-value problem $\mathbf{z}\left(\mathbf{t}_{k}\right)=\mathbf{x}\left(\mathbf{t}_{k}\right), \dot{\mathbf{z}}=f_{\mathbf{q}\left(t_{k}\right)}(\mathbf{z}), \forall t \geqslant \mathbf{t}_{k} ;$ and let $\left[\mathbf{t}_{k}, \mathbf{T}\right)$ denote its maximum interval of existence.

3". Draw independent random numbers $\mathbf{h}_{k}^{\ell}, \ell \in \mathcal{L}$ with standard exponential distributions (unit mean and variance).

4". If one of the integrals

$$
\mathbf{m}_{k}^{\ell}(t):=\int_{\mathbf{t}_{k}}^{t} \lambda_{\ell}\left(\mathbf{q}\left(\mathbf{t}_{k}\right), \mathbf{z}(s)\right) d s, \quad \forall t \in\left[\mathbf{t}_{k}, \mathbf{T}\right), \ell \in \mathcal{L}
$$

reaches $\mathbf{h}_{k}^{\ell}, \ell \in \mathcal{L}$ in the interval $\left(\mathbf{t}_{k}, \mathbf{T}\right)$, i.e., if

$$
\mathbf{t}_{k+1}:=\inf \left\{t \in\left(\mathbf{t}_{k}, \mathbf{T}\right): \exists \ell \in \mathcal{L}, \mathbf{m}_{k}^{\ell}(t) \geqslant \mathbf{h}_{k}^{\ell}\right\}
$$

belongs to the (open) interval $\left(\mathbf{t}_{k}, T\right)$ and $\exists \boldsymbol{\ell}_{k} \in \mathcal{L}$ such that $\mathbf{m}_{k}^{\boldsymbol{\ell}_{k}}(t) \geqslant \mathbf{h}_{k}^{\boldsymbol{\ell}_{k}}$; then define the SDSHS state in the interval $\left[\mathbf{t}_{k}, \mathbf{t}_{k+1}\right]$ according to (2)-(3). Increment the integer $k$ and go back to $2 "$.

5". Otherwise, define the SDSHS state in the interval $\left[\mathbf{t}_{k}, \mathbf{T}\right)$ according to (4) and terminate.

Here again, the random times $\mathbf{t}_{k}$ are called reset times and this solution is defined up to a maximal time $\mathbf{T}_{\max }$ that can be either equal to $\mathbf{T}$ if the construction terminate through 5 " or $\sup _{k} \mathbf{t}_{k}$ if it does not terminate.

The following conditions can be used to make sure that this construction succeeds with probability 1 and will be assumed throughout this paper.

Assumption 2 (SDSHS Standing Assumptions). The following is assumed for the SDSHSs considered throughout this paper:

A4 The vector fields $x \mapsto f_{q}(x), q \in \mathcal{Q}$ are locally Lipschitz.

A5 The transition intensity maps $x \mapsto \lambda_{\ell}(q, x), \ell \in \mathcal{L}, q \in \mathcal{Q}$ are continuous (or at least measurable).

A6 The transition intensities $\lambda_{\ell}(q, x), \ell \in \mathcal{L}$ and the reset maps $\phi_{\ell}(q, x), \ell \in \mathcal{L}$ are globally bounded in $q$ and locally bounded in $x$, in the sense that, for every bounded set $\mathcal{B} \subset \mathbb{R}^{n}$, there exists constants $L_{\mathcal{O}}, F_{\mathcal{O}}$ such that

$$
\lambda_{\ell}(q, x) \leqslant L_{\mathcal{O}}, \quad\left\|\phi_{\ell}(q, x)\right\| \leqslant F_{\mathcal{O}}, \quad, \forall q \in \mathcal{Q}, x \in \mathcal{B} .
$$

Assumption A4 makes sure that the initial-value problem in step 2" has a (local) solution and A5 makes sure that the integrals in (7) are well defined. Assumption A6 is not required for the construction of the solution, but it will be instrumental in establishing global existence of solutions. Specifically, A6 together with A4 allow us to conclude that, if the state $\mathbf{x}$ remains inside a bounded 
set, then $\mathbf{T}_{\max }=\infty^{2}$. Consequently, $\mathbf{T}_{\max }<\infty$ must mean that, in the interval $\left[0, \mathbf{T}_{\max }\right), \mathbf{x}(t)$ leaves any bounded set.

Finally, note that under this construction, the $\mathbf{h}_{k}^{\ell}, \ell \in \mathcal{L}$ are all positive with probability one and one does not have to worry about $\mathbf{t}_{k+1}$ in step 4 " being equal to $\mathbf{t}_{k}$. The probability of multiple $\mathbf{m}_{k}^{\ell}$ reaching the $\mathbf{h}_{k}^{\ell}$ simultaneously is also zero, so $\boldsymbol{\ell}_{k}$ in 4 " is well defined with probability one. In view of this, we do not need to worry about this construction failing to produce cadlag signals for $\mathbf{x}$ and $\mathbf{q}$, which was a concern in the constructions in Sections 2.1 and 2.2.

Example 2 (Estimation through a packet-switched network). Figure 7 depicts a scenario where one wants to construct a remote estimate for the state of a process. This state is measured by sensors that transmit their measurements to a remote location through a packet-switched shared network. Our goal is to accurately reconstruct the state of the process by sending a relatively small number of messages through the network. Inspired by [5, 9, 18, 26, 28], we propose a stochastic rule to send data that increases the rate at which data is sent as the state estimation error grows.

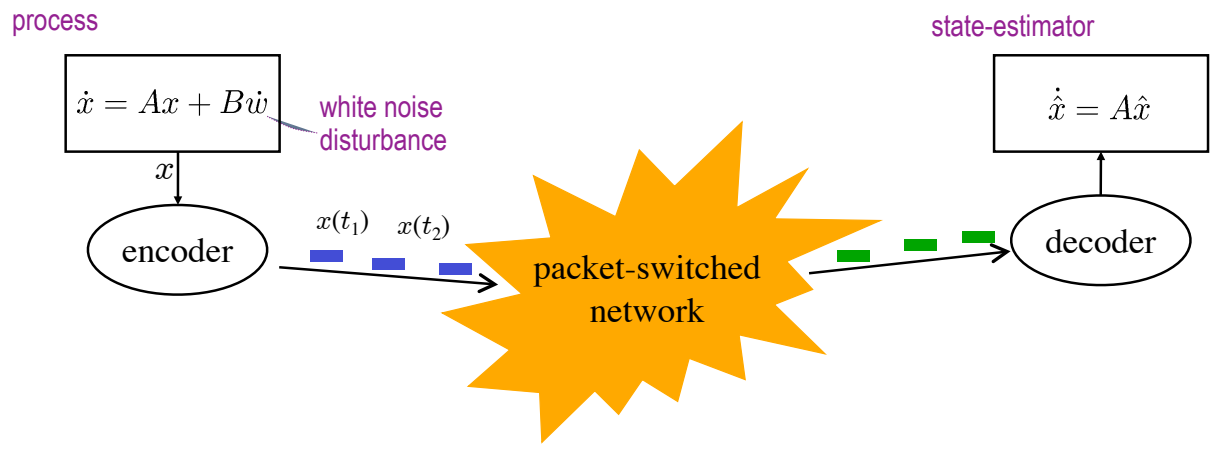

Figure 7: Estimation through a packet-switched network in Example 2.

Figure 7 shows the process to be an LTI system driven by a white noise process of the form

$$
\dot{\mathbf{x}}=A \mathbf{x}+b \dot{\mathbf{w}}, \quad \mathbf{x} \in \mathbb{R}^{n},
$$

where $\mathbf{w}$ denotes a scalar Brownian motion process. However, in this paper we want to focus our attention on SHSs whose dynamics in each mode are given by Ordinary Differential Equations (ODEs) and not by Stochastic Differential Equations (SDEs), since the former are somewhat simpler

\footnotetext{
${ }^{2}$ If the state $\mathbf{x}$ remains inside a bounded set $\mathcal{B}$, then $\mathrm{A} 4$ precludes the existence of finite escape time. In addition, A6 precludes the reset times $\mathbf{t}_{k}$ to have a finite accumulation points. Indeed, to have $\mathbf{t}_{k+1}$ finite, we must have

$$
\mathbf{m}_{k}^{\ell}\left(\mathbf{t}_{k+1}\right)=\int_{\mathbf{t}_{k}}^{\mathbf{t}_{k+1}} \lambda_{\ell}\left(\mathbf{q}\left(\mathbf{t}_{k}\right), \mathbf{x}(s)\right) d s \geqslant \mathbf{h}_{k}^{\ell}
$$
}

for some $\ell \in \mathcal{L}$. But if $\lambda_{\ell}\left(\mathbf{q}\left(\mathbf{t}_{k}\right), \mathbf{x}(s)\right)$ is upper bounded by $L_{\mathcal{O}}$, this implies that $L_{\mathcal{O}}\left(\mathbf{t}_{k+1}-\mathbf{t}_{k}\right) \geqslant \mathbf{h}_{k}^{\ell}$. Having a finite accumulation point for the $\mathbf{t}_{k}$ would thus require

$$
\frac{1}{L_{\mathcal{O}}} \sum_{k=0}^{\infty} \mathbf{h}_{k}^{\ell} \leqslant \sum_{k=0}^{\infty}\left(\mathbf{t}_{k+1}-\mathbf{t}_{k}\right)<\infty \Rightarrow \sum_{k=0}^{\infty} \mathbf{h}_{k}^{\ell}<\infty,
$$

which is a zero probability event since the $\mathbf{h}_{k}^{\ell}$ are i.i.d. random variables with standard exponential distributions. 
from a technical perspective. We will thus employ the mathematical trick of approximating an SDE by a random walk that can be easily modeled by an ODE with stochastic impulses: It turns out that the solutions to (8) can be obtained as the limit $\epsilon \downarrow 0^{+}$of the solutions to the SDSHS of a SHS with continuous dynamics

$$
\dot{\mathbf{x}}=A \mathbf{x}
$$

and two reset maps

$$
\phi_{1}(x):=x+\sqrt{\epsilon} b, \quad \quad \phi_{2}(x):=x-\sqrt{\epsilon} b, \quad \forall x \in \mathbb{R}^{n},
$$

both with the same transition intensities

$$
\lambda_{1}(x)=\lambda_{2}(x):=\frac{1}{2 \epsilon}, \quad \forall x \in \mathbb{R}^{n} .
$$

It is often the case that this SDSHS model is close to the physical mechanisms that are used to justify the idealized white noise process. While the idealized white noise process is often mathematically very convenient, we shall see shortly that this non-idealized noise process can still be simple to analyze.

The sensor/encoder in Figure 7 samples the state of the system at random times $\mathbf{t}_{k}, k \in \mathbb{Z}_{\geqslant 0}$ and sends the sampled states $\mathbf{x}\left(\mathbf{t}_{k}\right)$ to a decoder that builds the remote estimate $\hat{\mathbf{x}}$ of the true state $\mathbf{x}$. Between sample times, the decoder uses an open-loop state estimator of the form

$$
\dot{\hat{\mathbf{x}}}=A \hat{\mathbf{x}}, \quad \forall t \in\left[\mathbf{t}_{k}, \mathbf{t}_{k+1}\right),
$$

and, whenever a sample $\mathbf{x}\left(\mathbf{t}_{k}\right)$ is received, it resets the estimate $\hat{\mathbf{x}}\left(\mathbf{t}_{k}\right)$ to the value received. We consider here a scenario where the encoder generates the sample times $\mathbf{t}_{k}$ stochastically in a statedriven fashion. Specifically, we assume that these times are generated by a SDSHS model with a transition intensity that is a function of the state estimation error $\mathbf{e}:=\mathbf{x}-\hat{\mathbf{x}}$. This estimation error is available to the encoder because it can locally replicate the equations that the decoder uses to construct the estimate $\hat{\mathbf{x}}$ and it has direct access to the true state $\mathbf{x}$. Making the generation of sampling times that depend on the estimation error makes intuitive sense since one would expect that when the state estimation error at the decoder is very small, there is little gain in sending a state measurement. However, when the error is large, it is important to correct this error as fast as possible by sending a measurement. We shall see later that this intuition is indeed formally justifiable.

The error dynamics for the system described above can be summarized as follows: Between "event" times, the error evolves according to

$$
\dot{\mathbf{e}}=A \mathbf{e} .
$$

However, this dynamics is perturbed by two types of events: jumps in $\mathbf{x}$ due to the noise process (9) that lead to resets of the form

$$
\mathbf{e}\left(\mathbf{t}_{k}\right)=\phi_{1}\left(\mathbf{e}^{-}\left(\mathbf{t}_{k}\right)\right)=\mathbf{e}^{-}\left(\mathbf{t}_{k}\right)+\sqrt{\epsilon} b, \quad \mathbf{e}\left(\mathbf{t}_{k}\right)=\phi_{2}\left(\mathbf{e}^{-}\left(\mathbf{t}_{k}\right)\right)=\mathbf{e}^{-}\left(\mathbf{t}_{k}\right)-\sqrt{\epsilon} b,
$$

both with intensity $1 / 2 \epsilon$; and resets of the estimate $\hat{\mathbf{x}}$ due to the update of the estimate that leads to resets of the form

$$
\mathbf{e}\left(\mathbf{t}_{k}\right)=\phi_{3}\left(\mathbf{e}^{-}\left(\mathbf{t}_{k}\right)\right):=0
$$


with an intensity that typically depends on the current state estimation error e. These error dynamics can thus be represented by the SDSHS in Figure 8. This system has a single discrete mode and three reset maps, two to model the noise process and a third one to model the sampling of the state.

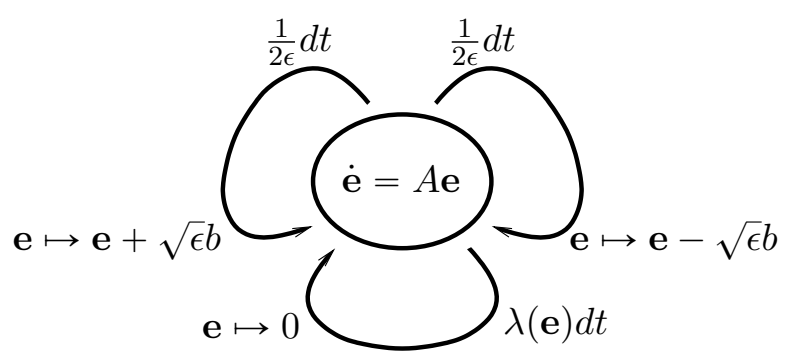

Figure 8: State-Driven Stochastic Hybrid System (SDSHS) that models the networked control system depicted in Example 2.

The system depicted in Figure 7 and consequently the model in Figure 8, make several restrictive simplification: we assume full state measurements for the encoder, measurement noise is ignored, quantization of the messages sent by the encoder is ignored, and it is assumed that the network does not introduce any delay. These simplifications are not particularly critical for the analysis that will follow and were made mostly for simplicity. From now let us briefly mention that if the encoder did not have access to the full state or if there were measurement errors, the encoder could incorporate a Kalman filter and send to the decoder, not the state of the process, but the state of the Kalman filter which "optimally" encodes all the information available up to the current time. Quantization of the values sent by the encoder essentially leads to replacing the reset to zero of the estimation error in (10) to a reset of the estimation error to the value of the quantization error. Finally, one could model the network delays by adding an additional state variable that keeps track of messages that are "in route" from encoder to decoder and make a distinction between events corresponding to the times at which the state is sampled and the times at which the corresponding message arrives at the decoder.

\subsection{Time-Triggered Stochastic Hybrid Systems as State-Driven Stochastic Hy- brid Systems}

While TTSHSs may seem to be fundamentally different from SDSHS, TTSHSs can be emulated by appropriately constructed SDSHSs when the reset-time distributions have probability density functions, i.e., when there exist functions $f_{q}^{\ell}:[0, \infty) \rightarrow[0, \infty)$ such that

$$
F_{q}^{\ell}(h):=\int_{[0, h]} \mu_{q}^{\ell}(d s)=\int_{0}^{h} f_{q}^{\ell}(s) d s, \quad \forall h \in\left[0, T_{q}^{\ell}\right), q \in \mathcal{Q}, \ell \in \mathcal{L},
$$

where $T_{q}^{\ell}:=\sup \left\{h \in \mathbb{R}: F_{q}^{\ell}(h)<1\right\} \in \mathbb{R} \cup\{\infty\}$. In this case, we can construct a SDSHS that emulates the TTSHS using the following procedure: We start by augmenting the continuous state of the TTSHS with a timer variable

$$
\boldsymbol{\tau}(t)=t-\mathbf{t}_{k}, \quad \forall t \geqslant 0
$$


leading to a SDSHS with continuous state $(\mathbf{x}, \boldsymbol{\tau}) \in \mathbb{R}^{n} \times \mathbb{R}$. The vector field for the state $\boldsymbol{\tau}$ is simply

$$
\dot{\tau}=1
$$

and the SDSHS reset maps $\phi_{\ell}: \mathcal{Q} \times \mathbb{R}^{n} \times \mathbb{R} \rightarrow \mathcal{Q} \times \mathbb{R}^{n} \times \mathbb{R}$ should enforce a reset of $\boldsymbol{\tau}$ to 0 at each reset time $\mathbf{t}_{k}$, which leads precisely to (11). The SDSHS should then have transitions intensities $\lambda_{\ell}(q, x, \tau)$ given by the hazard rate of the reset-time distribution $\mu_{q}^{\ell}$ :

$$
\lambda_{\ell}(q, x, \tau):=\frac{f_{q}^{\ell}(\tau)}{1-F_{q}^{\ell}(\tau)}, \quad \forall q \in \mathcal{Q}, x \in \mathbb{R}^{n}, \tau \in\left[0, T_{q}^{\ell}\right),
$$

which essentially provides the probability that a random timer $\tau$ with distribution $\mu_{q}^{\ell}$ will fire in an elementary interval $[\tau, \tau+d \tau)$, given that it has not yet fired at time $\tau$ :

$$
\lim _{d \tau \rightarrow 0} \frac{\mathrm{P}_{\mu_{q}^{\ell}}(\tau \leqslant \boldsymbol{\tau}<\tau+d \tau \mid \boldsymbol{\tau}>\tau)}{d \tau}=\frac{f_{q}^{\ell}(\tau)}{1-F_{q}^{\ell}(\tau)} .
$$

To understand why this works, we go back to the construction of the solution to the TTSHS in Section 2.2, in which the reset time $\mathbf{t}_{k+1}$ is determined by

$$
\mathbf{t}_{k+1}=\mathbf{t}_{k}+\min _{\ell \in \mathcal{L}} \mathbf{h}_{k}^{\ell}
$$

where the $\mathbf{h}_{k}^{\ell}$ are independent random variables with distributions $\mu_{\mathbf{q}\left(\mathbf{t}_{k}\right)}^{\ell}$. Therefore, the (conditional) distribution of $\mathbf{t}_{k+1}$ given $\mathbf{t}_{k}$ and $\mathbf{q}\left(\mathbf{t}_{k}\right)$ is determined by

$$
\mathrm{P}\left(\mathbf{t}_{k+1}>T \mid \mathbf{t}_{k}, \mathbf{q}\left(\mathbf{t}_{k}\right)\right)=\mathrm{P}\left(\mathbf{h}_{k}^{\ell}>T-\mathbf{t}_{k}, \forall \ell \in \mathcal{L} \mid \mathbf{t}_{k}, \mathbf{q}\left(\mathbf{t}_{k}\right)\right)=\prod_{\ell \in \mathcal{L}} \int_{\left(T-\mathbf{t}_{k}, \infty\right)} f_{\mathbf{q}\left(\mathbf{t}_{k}\right)}^{\ell}(s) d s .
$$

In contrast, in a SDSHS the reset time $\mathbf{t}_{k+1}$ is determined by

$$
\mathbf{t}_{k+1}:=\inf \left\{t \in\left[\mathbf{t}_{k}, \mathbf{T}\right): \exists \ell \in \mathcal{L}, \int_{\mathbf{t}_{k}}^{t} \lambda_{\ell}\left(\mathbf{q}\left(\mathbf{t}_{k}\right), \mathbf{z}(s)\right) d s \geqslant \mathbf{h}_{k}^{\ell}\right\}
$$

where where $\mathbf{z}(s)$ denotes the solution of the continuous vector field initialized at the value of the state at time $\mathbf{t}_{k}$, and the $\mathbf{h}_{k}^{\ell}, \ell \in \mathcal{L}$ are independent random variables with standard exponential distributions. Therefore, the (conditional) distribution of $\mathbf{t}_{k+1}$ given $\mathbf{t}_{k}, \mathbf{q}\left(\mathbf{t}_{k}\right), \mathbf{x}\left(\mathbf{t}_{k}\right)$ is now determined by

$$
\begin{aligned}
\mathrm{P}\left(\mathbf{t}_{k+1}>T \mid \mathbf{t}_{k}, \mathbf{q}\left(\mathbf{t}_{k}\right), \mathbf{x}\left(\mathbf{t}_{k}\right)\right) & =\mathrm{P}\left(\int_{\mathbf{t}_{k}}^{T} \lambda_{\ell}\left(\mathbf{q}\left(\mathbf{t}_{k}\right), \mathbf{z}(s)\right) d s<\mathbf{h}_{k}^{\ell}, \forall \ell \in \mathcal{L} \mid \mathbf{t}_{k}, \mathbf{q}\left(\mathbf{t}_{k}\right), \mathbf{x}\left(\mathbf{t}_{k}\right)\right) \\
& =\prod_{\ell \in \mathcal{L}} e^{-\int_{\mathbf{t}_{k}}^{T} \lambda_{\ell}\left(\mathbf{q}\left(\mathbf{t}_{k}\right), \mathbf{z}(s)\right) d s}
\end{aligned}
$$

The above construction for the SDSHS guarantees that

$$
\lambda_{\ell}\left(\mathbf{q}\left(\mathbf{t}_{k}\right), \mathbf{z}\left(\mathbf{t}_{k}+\tau\right)\right)=\frac{f_{\mathbf{q}\left(\mathbf{t}_{k}\right)}^{\ell}(\tau)}{1-F_{\mathbf{q}\left(\mathbf{t}_{k}\right)}^{\ell}(\tau)}, \quad \forall \tau \in\left[0, T_{\mathbf{q}\left(\mathbf{t}_{k}\right)}^{\ell}\right),
$$


and therefore

$$
\begin{aligned}
-\int_{\mathbf{t}_{k}}^{T} \lambda_{\ell}\left(\mathbf{q}\left(\mathbf{t}_{k}\right), \mathbf{z}(s)\right) d s=-\int_{0}^{T-\mathbf{t}_{k}} \lambda_{\ell}\left(\mathbf{q}\left(\mathbf{t}_{k}\right), \mathbf{z}\left(\mathbf{t}_{k}+\tau\right)\right) d \tau=\int_{0}^{T-\mathbf{t}_{k}} \frac{-f_{\mathbf{q}\left(\mathbf{t}_{k}\right)}^{\ell}(\tau)}{1-F_{\mathbf{q}\left(\mathbf{t}_{k}\right)}^{\ell}(\tau)} d \tau \\
=\int_{0}^{T-\mathbf{t}_{k}} \frac{-F_{\mathbf{q}\left(\mathbf{t}_{k}\right)}^{\ell}(\tau)^{\prime}}{1-F_{\mathbf{q}\left(\mathbf{t}_{k}\right)}^{\ell}(\tau)} d \tau=\left[\log \left(1-F_{\mathbf{q}\left(\mathbf{t}_{k}\right)}^{\ell}(\tau)\right)\right]_{0}^{T-\mathbf{t}_{k}}=\log \left(1-F_{\mathbf{q}\left(\mathbf{t}_{k}\right)}^{\ell}(T)\right),
\end{aligned}
$$

which shows a match between the two conditions (12) and (13) that determine the resets of the TTSHS and the SDSHS, respectively.

This ability to represent TTSHS as SDSHS, enables one to combine in the same SHS both time-triggered and state-driven resets. However, this can only be done, as shown here, when the reset-time distributions have probability density functions and therefore do not have point masses.

\section{Stability of SHSs}

To characterize the stability of a stochastic process one may pursue two alternative types of conditions: One is focused on making sure that the probability that the stochastic process "misbehaves" is very small. Such notions are often called sample-path stability notions because they address the behavior of individual sample paths of the stochastic process. Alternatively, one may focus ones attention on aggregate behavior and require that the process "behaves" well on the average. Such notions are called mean or aggregate notions of stability.

We start by considering sample path notions of stability that attempt to parallel the key concepts behind Lyapunov stability for deterministic systems. Sample-path stability notions are typically qualified by the suffix "in probability." A SHS (either time-triggered or state-driven) with discrete state $\mathbf{q}:\left[0, \mathbf{T}_{\max }\right) \rightarrow \mathcal{Q}$ and continuous state $\mathbf{x}:\left[0, \mathbf{T}_{\max }\right) \rightarrow \mathbb{R}^{n}$ is said to be

D1 (Lyapunov) stable in probability if it is globally defined with probability one, i.e., $\mathbf{T}_{\max } \stackrel{\text { wpo }}{=} \infty$ and, for every $\epsilon, \rho>0$, there exists a $\delta>0$ such that, for every initial condition $\mathbf{q}(0)=q_{0} \in \mathcal{Q}$, $\mathbf{x}(0)=x_{0} \in \mathbb{R}^{n}$,

$$
\left\|x_{0}\right\| \leqslant \delta \quad \Rightarrow \quad \mathrm{P}(\exists t \in[0, \infty):\|\mathbf{x}(t)\|>\rho) \leqslant \epsilon
$$

D2 (Lyapunov) asymptotically stable in probability if it is (Lyapunov) stable in probability and $\mathbf{x}(t)$ converges to zero with probability one, i.e.,

$$
\mathrm{P}\left(\lim _{t \rightarrow \infty} \mathbf{x}(t)=0\right)=1
$$

Aggregate or mean notions of stability require the selection of a non-negative function $W: \mathcal{Q} \times \mathbb{R}^{n} \rightarrow$ $[0, \infty)$ and the SHS is said to be

D3 mean-W stable if it is globally defined with probability one, i.e., $\mathbf{T}_{\max } \stackrel{\text { wpo }}{=} \infty$ and, for every initial condition $\mathbf{q}(0)=q_{0} \in \mathcal{Q}, \mathbf{x}(0)=x_{0} \in \mathbb{R}^{n}$,

$$
\mathrm{E}[W(\mathbf{q}(t), \mathbf{x}(t))]<\infty, \quad \forall t \geqslant 0
$$


D4 stochastically mean- $W$ stable if it is globally defined with probability one, i.e., $\mathbf{T}_{\max } \stackrel{\text { wpo }}{=} \infty$ and, for every initial condition $\mathbf{q}(0)=q_{0} \in \mathcal{Q}, \mathbf{x}(0)=x_{0} \in \mathbb{R}^{n}$,

$$
\int_{0}^{\infty} \mathrm{E}[W(\mathbf{q}(t), \mathbf{x}(t))] d t<\infty
$$

D5 asymptotically mean- $W$ stable if it is globally defined with probability one, i.e., $\mathbf{T}_{\max } \stackrel{\text { wpo }}{=} \infty$ and, for every initial condition $\mathbf{q}(0)=q_{0} \in \mathcal{Q}, \mathbf{x}(0)=x_{0} \in \mathbb{R}^{n}$,

$$
\lim _{t \rightarrow \infty} \mathrm{E}[W(\mathbf{q}(t), \mathbf{x}(t))]=0
$$

D6 exponentially mean- $W$ stable if it is globally defined with probability one, i.e., $\mathbf{T}_{\max } \stackrel{\text { wpo }}{=} \infty$ and there exist constants $c, \lambda>0$ such that, for every initial condition $\mathbf{q}(0)=q_{0} \in \mathcal{Q}$, $\mathbf{x}(0)=x_{0} \in \mathbb{R}^{n}$,

$$
\mathrm{E}[W(\mathbf{q}(t), \mathbf{x}(t))] \leqslant c e^{-\lambda t} W\left(q_{0}, x_{0}\right), \quad \forall t \geqslant 0
$$

When the function $W$ is the squared Euclidean norm of the continuous state (i.e., $W(q, x)=\|x\|^{2}$, $\forall q \in \mathcal{Q}, x \in \mathbb{R}^{n}$ ) we simply say that the processes are mean-square stable, without reference to $W$.

\section{Analysis of Time-Triggered SHSs}

While the stability analysis of general SHSs is challenging, very complete results appeared recently for the class of linear TTSHSs. These are TTSHSs for which the vector fields and reset maps are both linear functions of the state. To present these results, we consider the simpler case of the impulsive system in Figure 9, with a single linear vector field

$$
\dot{\mathbf{x}}=A \mathbf{x},
$$

and a single linear reset map

$$
\phi(x):=J x, \quad \forall x \in \mathbb{R}^{n}
$$

associated with a reset-time distribution $\mu$ for the inter-reset intervals $\mathbf{h}_{k}:=\mathbf{t}_{k+1}-\mathbf{t}_{k}$.

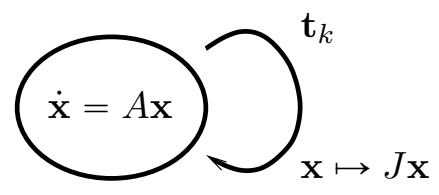

Figure 9: Linear Time-Triggered Stochastic Impulsive System

To study the stability of the linear time-triggered impulsive systems (TTSIS) in Figure 9, it is convenient to define a (stochastic) discrete-time system obtained by sampling the state $\mathbf{x}$ at the reset times $\mathbf{t}_{k}$ :

$$
\mathbf{x}_{k}:=\mathbf{x}\left(\mathbf{t}_{k}\right), \quad \forall k \in \mathbb{Z}_{\geqslant 0}
$$


The dynamics of this discrete-time system can be easily derived and are given by

$$
\mathbf{x}_{k+1}=J e^{A\left(\mathbf{t}_{k+1}-\mathbf{t}_{k}\right)} \mathbf{x}_{k}=J e^{A \mathbf{h}_{k}} \mathbf{x}_{k}, \quad \forall k \in \mathbb{Z}_{\geqslant 0},
$$

and therefore, for a given positive definite matrix $P=P^{\prime}>0$, we can compute

$$
\mathrm{E}\left[\mathbf{x}_{k+1}^{\prime} P \mathbf{x}_{k+1} \mid \mathbf{x}_{k}\right]=\mathbf{x}_{k} \mathrm{E}\left[e^{A^{\prime} \mathbf{h}_{k}} J^{\prime} P J e^{A \mathbf{h}_{k}}\right] \mathbf{x}_{k},
$$

where we used the fact that value of the state $\mathbf{x}_{k}$ at time $\mathbf{t}_{k}$ is independent of the interval $\mathbf{h}_{k}:=$ $\mathbf{t}_{k+1}-\mathbf{t}_{k}, \forall k \in \mathbb{Z}_{\geqslant 0}$. Suppose now that we can find a positive definite matrix $P$ for which

$$
\mathrm{E}\left[e^{A^{\prime} \mathbf{h}_{k}} J^{\prime} P J e^{A \mathbf{h}_{k}}\right]=\int_{0}^{\infty} e^{A^{\prime} h} J^{\prime} P J e^{A h} \mu(d h) \leqslant \gamma P,
$$

for some $\gamma \in(0,1)$, where the expected value is taken with respect to the random variable $\mathbf{h}_{k}$ whose distribution is defined by the reset-time distribution $\mu$. In this case, we conclude from (18) and (19) that

$$
\mathrm{E}\left[\mathbf{x}_{k+1}^{\prime} P \mathbf{x}_{k+1} \mid \mathbf{x}_{k}\right] \leqslant \gamma \mathbf{x}_{k} P \mathbf{x}_{k} .
$$

Taking expected values on both sides of the above inequality, we would then conclude that

$$
\mathrm{E}\left[\mathbf{x}_{k+1}^{\prime} P \mathbf{x}_{k+1}\right] \leqslant \gamma \mathrm{E}\left[\mathbf{x}_{k}^{\prime} P \mathbf{x}_{k}\right]
$$

from which it would be straightforward to show that $\mathrm{E}\left[\left\|\mathbf{x}_{k}\right\|^{2}\right]$ converges (exponentially fast) to zero as $k \rightarrow \infty$. It turns out that the stability of the TTSIS does not always follow from this because of the behavior of $\mathbf{x}(t)$ between reset times. In particular, problems could arise if (i) $A$ is unstable, and therefore $\mathbf{x}(t)$ tends to grow exponentially between reset times; and (ii) the distribution of $\mathbf{h}_{k}$ has infinite support, and therefore the interval between reset times can be arbitrarily large (albeit with low probability). Nevertheless, by adding appropriate conditions to address the inter-reset behavior of $\mathbf{x}$, we can express fairly simple necessary and sufficient condition for the stability of the TTSIS. It turns out that necessary and sufficient conditions for the stability of a TTSIS depend on the type of stochastic stability considered, as expressed by the result below.

Theorem 1 (Stability of a Linear TTSIS [2]). Consider a TTSIS defined by the vector field (15), the reset map (16), and the reset-time distribution $\mu$, for which Assumption 1 holds. Further assume that

$$
\sigma\left(\sum_{i} \mu\left(\left\{b_{i}\right\}\right)\left(J e^{A b_{i}}\right)^{\prime} \otimes\left(J e^{A b_{i}}\right)\right)<1, \quad \exists \lambda>\lambda^{*}: \quad \int_{0}^{\infty} e^{2 \lambda h} \mu(d h)<\infty,
$$

where $\sigma(M)$ denotes the spectral radius of the matrix $M$, the summation inside $\sigma(\cdot)$ is taken over any point masses $b_{i}$ of the reset-time distribution $\mu$, and $\lambda^{*}$ denotes the real part of the eigenvalue of $A$ with largest real part for which one of the corresponding generalized eigenvectors is not in the kernel of $J$, i.e.,

$$
\lambda^{*}:=\max \left\{\Re[\lambda]:(A-\lambda I)^{k} x=0, J x \neq 0, k \in \mathbb{Z}_{\geqslant 1}, x \in \mathbb{R}^{n}, \lambda \in \mathbb{C}\right\} .
$$


P1 This system is stochastically mean-square stable (Definition D4 with squared norm $W$ ) if and only if there exists a positive definite matrix $P=P^{\prime}>0$ such that

$$
L(P)<P, \quad L(P):=\int_{0}^{\infty} e^{A^{\prime} h} J^{\prime} P J e^{A h} \mu(d h),
$$

and

$$
\int_{0}^{\infty} h^{2\left(m_{\Re}(A)-1\right)} e^{2 \lambda_{\Re}(A) h} r(h) d h<\infty,
$$

where $\lambda_{\Re}(A)$ denotes the real part of the eigenvalue of $A$ with largest real part, $m_{\Re}$ the dimension of the corresponding Jordan block, and $r(h):=\mathrm{P}\left(\mathbf{h}_{k}>h\right)=\int_{(h, \infty)} \mu(d h)$ denotes the survivor function of $\mu$.

P2 This system is asymptotically mean-square stable (Definition D5 with squared norm W) if and only if there exists a positive definite matrix $P=P^{\prime}>0$ such that (20) holds and

$$
\lim _{h \rightarrow \infty} h^{2\left(m_{\Re}(A)-1\right)} e^{2 \lambda_{\Re}(A) h} r(h)=0 .
$$

P3 This system is exponentially mean-square stable (Definition D6 with squared norm $W$ ) if and only if there exists a positive definite matrix $P=P^{\prime}>0$ such that (20) holds and there exist constants $c, \alpha>0$ such that

$$
h^{2\left(m_{\Re}(A)-1\right)} e^{2 \lambda_{\Re}(A) h} r(h) \leqslant c e^{-\alpha t}, \quad \forall h \geqslant 0 .
$$

We can see from Theorem 1 that the existence of the positive definite matrix $P=P^{\prime}>0$ such that (20) holds is necessary for all three types of stability. The reader can easily verify that this condition is equivalent to the condition that we encountered in (19), and that enabled us to show that the sampled state (17) converges to zero exponentially fast as $k \rightarrow \infty$. However, we can now see that, in general, this does not suffice to prove stability. The conditions (21), (22), and (23) are needed to make sure that the state remains "well-behaved" between reset times. It turns out that all these three conditions hold trivially if the matrix $A$ is Hurwitz or if the distribution of the $\mathbf{h}_{k}$ have finite support (i.e., if $\exists h>0$ such that $\left.r(h):=\mathrm{P}\left(\mathbf{h}_{k}>h\right)=0\right)$. For unstable matrices $A$ and distributions with infinite support, stability essentially depends on how the rate of growth of $e^{A t}$ compares with the survival function $r(h)$ of the reset-time distribution $\mu$.

From a computational perspective, the condition (20) is a Linear Matrix Inequality since the map $P \mapsto L(P)$ is linear. The existence of the matrix $P$ can thus be easily verified numerically. This same condition can also be verified by computing the spectral value of an appropriately defined matrix or through the evaluation of a Nyquist contour [2]. The conditions (21), (22), and (23) are also easy to verify as they simply depend on the "least stable" eigenvalue of $A$.

While the sufficiency of the conditions in Theorem 1 is fairly easy to establish based on the reasoning that we started to outline above, their necessity requires a more sophisticated proof that can be found in [2]. In addition, the reader is referred to [1] for results pertaining to multiple resets and to [4] for multiple resets and multiple discrete modes. In these references, the reader can also find numerical examples illustrating how these results can be used to analyze NCSs analogous to the one described in Example 1. 


\section{Analysis of State-Driven SHSs}

Lyapunov-based methods provide a very versatile set of tools that can be used to establish the stability of (deterministic) Ordinary Differential Equations (ODEs) like

$$
\dot{x}=f(x), \quad x \in \mathbb{R}^{n} .
$$

A key enabling tool for Lyapunov-based methods is the chain-rule for taking derivative, which enable us to compute the time-derivative of a function along trajectories of the ODE. Specifically, given a continuously differentiable function $V: \mathbb{R}^{n} \rightarrow \mathbb{R}$, we have that

$$
\frac{d V(x(t))}{d t}=\frac{\partial V(x(t))}{\partial x} \frac{d x(t)}{d t}=\frac{\partial V(x(t))}{\partial x} f(x(t))
$$

for $t$ in any interval $[0, T)$ on which the solution to (24) is defined and continuously differentiable. This result enables one to conclude that the algebraic condition

$$
\frac{\partial V(x)}{\partial x} f(x) \leqslant 0, \quad \forall x \in \mathbb{R}^{n}
$$

suffices to conclude that the time function $t \mapsto V(x(t))$ is monotone non-increasing along solutions to (24), even if one is unable to explicitly compute such solutions. To extend this type of tools to SDSHSs we need something like the chain rule for this type of SHSs. Fortunately, such rule exists.

\subsection{Extended Generator}

Consider the SDSHS defined in Section 2.3. We define the extended generator of this SDSHS to be the operator $L$ that maps a continuously differentiable function $V: \mathcal{Q} \times \mathbb{R}^{n} \rightarrow \mathbb{R}$ into another function $L V: \mathcal{Q} \times \mathbb{R}^{n} \rightarrow \mathbb{R}$ according to the rule:

$$
L V(q, x):=\frac{\partial V(q, x)}{\partial x} f_{q}(x)+\sum_{\ell \in \mathcal{L}} \lambda_{\ell}(q, x)\left(V\left(\phi_{\ell}(q, x)\right)-V(q, x)\right), \quad \forall q \in \mathcal{Q}, x \in \mathbb{R}^{n} .
$$

It turns out that, under appropriate assumptions, one can use the extended generator to compute derivatives along solutions to the SDSHS. In particular,

$$
\begin{aligned}
& \mathrm{E}[V(\mathbf{q}(t), \mathbf{x}(t)) \mid \mathbf{q}(\tau), \mathbf{x}(\tau)] \stackrel{\text { wpo }}{=} \\
& \qquad(\mathbf{q}(\tau), \mathbf{x}(\tau))+\int_{\tau}^{t} \mathrm{E}[L V(\mathbf{q}(s), \mathbf{x}(s)) \mid \mathbf{q}(\tau), \mathbf{x}(\tau)] d s, \quad \forall t \geqslant \tau \geqslant 0,
\end{aligned}
$$

where $\stackrel{\text { wpo }}{=}$ refers to the fact that this equality holds with probability one. In the Markov processes' literature, (25) is often known as Dynkin's formula. This formula allows us to conclude from the Fundamental Theorem of Calculus for Lebesgue Integrals [12, p. 102] that $t \mapsto \mathrm{E}[V(\mathbf{q}(t), \mathbf{x}(t)) \mid \mathbf{q}(\tau), \mathbf{x}(\tau)]$ is absolutely continuous and differentiable almost everywhere, with

$$
\frac{d \mathrm{E}[V(\mathbf{q}(t), \mathbf{x}(t)) \mid \mathbf{q}(\tau), \mathbf{x}(\tau)]}{d t} \stackrel{\text { wpo }}{=} \mathrm{E}[L V(\mathbf{q}(t), \mathbf{x}(t)) \mid \mathbf{q}(\tau), \mathbf{x}(\tau)], \quad \forall t \stackrel{\text { ae }}{\in}[\tau, \infty), \forall \tau \geqslant 0,
$$

where the $\stackrel{\text { ae }}{\in}$ refers to the fact that the derivative may not exist on a set of times $t$ with zero measure. It is important to note that, even though the sample paths of the stochastic process $V(\mathbf{q}(t), \mathbf{x}(t))$ 
are typically discontinuous because of the resets, the expected value of this stochastic process is not only continuous but, in fact, differentiable almost everywhere. We shall refer to (25) as the differential form of Dynkin's formula.

Either form of the Dynkin's formula (for $\tau=0$ ) allow us to conclude that an algebraic condition of the form

$$
L V(q, x) \leqslant 0, \quad \forall q \in \mathcal{Q}, x \in \mathbb{R}^{n}
$$

suffices to establish that the time function $t \mapsto \mathrm{E}[V(\mathbf{q}(t), \mathbf{x}(t))]$ is upper bounded by $V\left(q_{0}, x_{0}\right)$ with probability one, which is the basis to establish stability for SDSHSs, even if one is unable to explicitly compute its solutions or the expectations that appear in (25) or (26).

A technical but important issue in applying Dynkin's formula is whether or not (25) holds for a particular function $V: \mathcal{Q} \times \mathbb{R}^{n} \rightarrow \mathbb{R}$. The following result adapted from [11] provides sufficient conditions for the validity of Dynkin's formulas.

Theorem 2. Consider the SDSHS defined in Section 2.3 and a continuously differentiable function $V: \mathcal{Q} \times \mathbb{R}^{n} \rightarrow \mathbb{R}$. Suppose that Assumption 2 holds and, in addition, the following conditions also hold:

A7 The differential equations $\dot{z}=f_{q}(z), q \in \mathcal{Q}$ all have globally defined solutions for every initial condition $z(0)=z_{0} \in \mathbb{R}^{n}$.

A8 For every initial condition $\mathbf{q}(0)=q_{0} \in \mathcal{Q}, \mathbf{x}(0)=x_{0} \in \mathbb{R}^{n}$, we have that

$$
\mathrm{E}[\mathbf{N}(T)]<\infty, \quad \forall T \geqslant 0
$$

where the (cadlag) stochastic process $\mathbf{N}(T):=\max \left\{k \in \mathbb{Z}_{\geqslant 0}: \mathbf{t}_{k} \leqslant T\right\}$ counts the number of resets up to time $T$.

A9 For every initial condition $\mathbf{q}(0)=q_{0} \in \mathcal{Q}, \mathbf{x}(0)=x_{0} \in \mathbb{R}^{n}$, we have that

$$
\mathrm{E}\left[\sum_{k=0}^{\mathbf{N}(T)} \mid V\left(\phi_{\ell}\left(\mathbf{q}\left(\mathbf{t}_{k}\right), \mathbf{x}\left(\mathbf{t}_{k}\right)\right)-V\left(\mathbf{q}^{-}\left(\mathbf{t}_{k}\right), \mathbf{x}^{-}\left(\mathbf{t}_{k}\right)\right) \mid\right]<\infty, \quad \forall T \geqslant 0 .\right.
$$

Then the SDSHS state $\mathbf{z}:=(\mathbf{q}, \mathbf{x})$ is a strong Markov process for which (25) and (26) hold.

One should clarify that Theorem 2 requires the left-hand sides of (27) and (28) to be finite for every initial condition and every finite $T$, but it does not require the existence of a uniform bound over all initial conditions and times $T$.

We recall that a stochastic process $\mathbf{z}(t) \in \mathbb{R}^{n}, \forall t \in[0, \infty)$ is called a Markov process if, for every measurable set $\mathcal{A} \subset \mathbb{R}^{n}$,

$$
\mathrm{P}(\mathbf{z}(t) \in \mathcal{A} \mid \mathbf{z}(s), \forall s \leqslant \tau)=\mathrm{P}(\mathbf{z}(t) \in \mathcal{A} \mid \mathbf{z}(\tau)) \quad \forall t \geqslant \tau \geqslant 0 ;
$$

which means that the only information relevant to evaluating the behavior of the process beyond tome $\tau$ is $\mathbf{z}(\tau)$. The same process is a strong Markov process if (29) holds not just for deterministic times $t, \tau$, but also for stochastic times $\mathbf{t}, \boldsymbol{\tau}$ that are stopping times for the natural filtration of $\mathbf{z}$. 
We recall that a random variable $\mathbf{T}$ is a stopping time for the natural filtration of $\mathbf{z}$ if, for every $t \geqslant 0$, one can determine whether or not $\mathbf{T} \leqslant t$ by observing $\mathbf{z}(s), \forall s \leqslant t$. We shall encounter examples of stopping times shortly.

While essential, Assumptions A8-A9 are often difficult to verify directly and can be violated by apparently innocuous SDSHSs. Consider, e.g., a SDSHS with a single discrete mode and a scalar state $\mathbf{x} \in \mathbb{R}$ that evolves according to $\dot{\mathbf{x}}=0$ between transitions, a single (nonlocally Lipschitz) reset map $\phi_{1}(x):=x^{p}, \forall x \in \mathbb{R}$, and a constant transition intensity $\lambda_{1}(x):=\mu, \forall x \in \mathbb{R}$ for constants $p \geqslant 1$ and $\mu>0$. Given a time instant $t>0$, it is straightforward to conclude that $\mathbf{x}(t)=x_{0}^{p^{\mathbf{N}(t)}}$ and therefore

$$
\mathrm{E}[\mathbf{x}(t)]=\sum_{n=0}^{\infty} \frac{x_{0}^{p^{n}} \mu^{n} t^{n}}{n !} e^{-\mu t}
$$

It turns out that when $x_{0}>1$ the above series does not converge for any $t>0$ and therefore (25) does not hold for any $t>\tau:=0$. This does not contradict Theorem 2 because Assumption A9 does not hold. Problems may still arise when the reset maps are globally Lipschitz. For example, considering instead the globally Lipschitz reset map $\phi_{1}(x):=p x, \forall x \in \mathbb{R}$ and a linear transition intensity $\lambda_{1}(x)=x, \forall x \in \mathbb{R}$, it is possible to show that $\mathrm{E}[\mathbf{x}(t)]$ explodes in finite time and therefore (25) only holds on a finite time interval. For this system, neither A8 nor A9 holds.

\subsection{Stopped Processes}

As mentioned above, a key challenge in using Dynkin's formulas to prove stability of a SDSHS is that often it is not easy to establish that the assumptions of Theorem 2 hold. In particular, the differential equations may have finite escape time, which invalidates $A 7$; the number of resets in a finite interval could potentially be unbounded, which would invalidate A8; or the summation in (28) could be unbounded, which would invalidate A9. In fact, the goal of proving stability of a SDSHS is often precisely to establish that these types of "misbehavior" cannot occur. A technique that can be used to overcome this challenge goes back at least 50 years and consists of analyzing an auxiliar "stopped" process (see, e.g., [19]), for which it is easier to establish the validity of the Dynkin's formula, and from which one can still conclude stability of the SDSHS.

Given a set $\mathcal{O} \subset \mathcal{Q} \times \mathbb{R}^{n}$, we define the $\mathcal{O}$-stopping time to be the first time that the SDSHS's state $\mathbf{z}(t):=(\mathbf{q}(t), \mathbf{x}(t)), t \in\left[0, \mathbf{T}_{\max }\right)$ leaves $\mathcal{O}$. Specifically,

$$
\mathbf{T}_{\mathcal{O}}= \begin{cases}\mathbf{T}_{\max } & \text { if } \mathbf{z}(t) \in \mathcal{O}, \forall t \in\left[0, \mathbf{T}_{\max }\right) \\ \inf \left\{t \in\left[0, \mathbf{T}_{\text {max }}\right): \mathbf{z}(t) \notin \mathcal{O}\right\} & \text { otherwise. }\end{cases}
$$

All stopping times considered in this paper are defined using sets $\mathcal{O} \subset \mathcal{Q} \times \mathbb{R}^{n}$ that are

A10 open in $x$ in the sense that, for every $q \in \mathcal{Q}, \mathcal{O}_{q}:=\left\{x \in \mathbb{R}^{n}:(q, x) \in \mathcal{O}\right\} \subset \mathbb{R}^{n}$ is an open subset of $\mathbb{R}^{n}$; and

A11 uniformly bounded in $x$ in the sense that there exists a constant $C_{\mathcal{O}}$ such that $\|x\| \leqslant C_{\mathcal{O}}$, $\forall(q, x) \in \mathcal{O}$. 
Because the state $\mathbf{z}$ is right-continuous and $\mathbf{q}$ is piecewise constant, A10 guarantees that the infimum in the second branch of (30) is actually a minimum and therefore

$$
\mathbf{z}(t) \stackrel{\text { wpo }}{\in} \mathcal{O}, \quad \forall t \in\left[0, \mathbf{T}_{\mathcal{O}}\right), \quad \text { and } \quad \mathbf{z}(t) \stackrel{\text { wpo }}{\notin} \mathcal{O}, \quad \forall t \in\left[\mathbf{T}_{\mathcal{O}}, \mathbf{T}_{\max }\right),
$$

where the right-hand side condition only makes sense when $\mathbf{T}_{\mathcal{O}}<\mathbf{T}_{\max }$. Also, as discussed in Section 2.3, if the state $\mathbf{x}$ remains inside a bounded set and Assumption 2 holds, then $\mathbf{T}_{\max }=\infty$. Therefore, for sets $\mathcal{O}$ satisfying A11, we must have $\mathrm{P}\left(\mathbf{T}_{\mathcal{O}}=\mathbf{T}_{\max }, \mathbf{T}_{\max }<\infty\right)=0$. Under these assumptions, we can define the $\mathcal{O}$-stopped processes to be the stochastic processes

$$
\mathbf{x}_{\mathcal{O}}(t):=\left\{\begin{array}{ll}
\mathbf{x}(t) & t \in\left[0, \mathbf{T}_{\mathcal{O}}\right) \\
\mathbf{x}\left(\mathbf{T}_{\mathcal{O}}\right) & t \in\left[\mathbf{T}_{\mathcal{O}}, \infty\right), \mathbf{T}_{\mathcal{O}}<\mathbf{T}_{\max },
\end{array} \mathbf{q}_{\mathcal{O}}(t):=\left\{\begin{array}{ll}
\mathbf{q}(t) & t \in\left[0, \mathbf{T}_{\mathcal{O}}\right) \\
\mathbf{q}\left(\mathbf{T}_{\mathcal{O}}\right) & t \in\left[\mathbf{T}_{\mathcal{O}}, \infty\right), \mathbf{T}_{\mathcal{O}}<\mathbf{T}_{\max },
\end{array} .\right.\right.
$$

Note that, because $\mathrm{P}\left(\mathbf{T}_{\mathcal{O}}=\mathbf{T}_{\max }, \mathbf{T}_{\max }<\infty\right)=0$, we do not need to consider the case $t \in\left[\mathbf{T}_{\mathcal{O}}, \infty\right)$, with $\mathbf{T}_{\mathcal{O}}=\mathbf{T}_{\max }$.

Aside from the technicalities mentioned above, which are needed to make sure that the stopped processes are well-defined, the stopped processes $\left(\mathbf{q}_{\mathcal{O}}, \mathbf{x}_{\mathcal{O}}\right)$ are equal to the SDHSH state $(\mathbf{q}, \mathbf{x})$ until the first time $\mathbf{T}_{\mathcal{O}}$ that the state exits $\mathcal{O}$ and remain constant after that time.

\subsubsection{Extended Generator of the Stopped Processes}

It turns out that the stopped process $\mathbf{x}_{\mathcal{O}}$ also satisfies Dynkin's formula, but for an extended generator that is zero outside $\mathcal{O}$. Specifically,

$$
\begin{aligned}
\mathrm{E}\left[V\left(\mathbf{q}_{\mathcal{O}}(t), \mathbf{x}_{\mathcal{O}}(t)\right) \mid \mathbf{q}_{\mathcal{O}}(\tau), \mathbf{x}_{\mathcal{O}}(\tau)\right] \stackrel{\text { wpo }}{=} \\
V\left(\mathbf{q}_{\mathcal{O}}(\tau), \mathbf{x}_{\mathcal{O}}(\tau)\right)+\int_{\tau}^{t} \mathrm{E}\left[L_{\mathcal{O}} V\left(\mathbf{q}_{\mathcal{O}}(s), \mathbf{x}_{\mathcal{O}}(s)\right) \mid \mathbf{q}_{\mathcal{O}}(\tau), \mathbf{x}_{\mathcal{O}}(\tau)\right] d s, \quad \forall t \geqslant \tau \geqslant 0,
\end{aligned}
$$

and

$$
\begin{aligned}
\frac{d \mathrm{E}\left[V\left(\mathbf{q}_{\mathcal{O}}(t), \mathbf{x}_{\mathcal{O}}(t)\right) \mid \mathbf{q}_{\mathcal{O}}(\tau), \mathbf{x}_{\mathcal{O}}(\tau)\right]}{d t} \stackrel{\text { wpo }}{=} \\
\mathrm{E}\left[L_{\mathcal{O}} V\left(\mathbf{q}_{\mathcal{O}}(t), \mathbf{x}_{\mathcal{O}}(t)\right) \mid \mathbf{q}_{\mathcal{O}}(\tau), \mathbf{x}_{\mathcal{O}}(\tau)\right], \quad \forall t \stackrel{\text { ae }}{\in}[\tau, \infty), \tau \geqslant 0,
\end{aligned}
$$

where

$$
L_{\mathcal{O}} V(q, x):= \begin{cases}L V(q, x) & (q, x) \in \mathcal{O} \\ 0 & (q, x) \notin \mathcal{O}\end{cases}
$$

When A10-A11 hold, the assumptions needed for the validity of the Dynkin's formula (32) are much easier to verify than those in Theorem 3, as noted in the following result also adapted from [11]:

Theorem 3. Consider the SDSHS defined in Section 2.3, a set $\mathcal{O} \subset \mathcal{Q} \times \mathbb{R}^{n}$ that satisfies A10-A11, the associated $\mathcal{O}$-stopped processes, and a continuously differentiable function $V: \mathcal{Q} \times \mathbb{R}^{n} \rightarrow \mathbb{R}$. If Assumption 2 holds, then $\mathbf{z}_{\mathcal{O}}:=\left(\mathbf{q}_{\mathcal{O}}, \mathbf{x}_{\mathcal{O}}\right)$ is a strong Markov process for which (31) and (32) hold. 


\subsubsection{Convergence of the Stopped Processes}

The key technical result needed to use stopped processes to draw conclusions on the state of the SDSHS is that, when the solution to the SHSDS exists globally (i.e., $\mathbf{T}_{\max } \stackrel{\text { wpo }}{=} \infty$ ), the stopped processes $\mathbf{q}_{\mathcal{O}}$ and $\mathbf{x}_{\mathcal{O}}$ converge (pointwise in time) to the states $\mathbf{q}$ and $\mathbf{x}$ of the SDSHS, as we enlarge the set $\mathcal{O}$ to cover the whole $\mathcal{Q} \times \mathbb{R}^{n}$. This allows us to use Fatou's Lemma to establish bounds on probabilities or expected values involving the SDSHS state, from bounds involving the stopped processes.

Lemma 1. Consider the SDSHS defined in Section 2.3, a monotone sequence of sets $\left\{\mathcal{O}_{1} \subset \mathcal{O}_{2} \subset\right.$ $\left.\cdots \subset \mathcal{O}_{m} \subset \cdots\right\}$ that satisfy A10-A11 and for which $\bigcup_{m=1}^{\infty} \mathcal{O}_{m}=\mathcal{Q} \times \mathbb{R}^{n}$, and the associated $\mathcal{O}_{m}$-stopped processes. If Assumption 2 holds and that the solution to the SDSHS exists globally with probability one [i.e., $\mathrm{P}\left(\mathbf{T}_{\max }=\infty\right)=1$ ], then:

$\mathbf{P} 4$ For every finite time $t \geqslant 0$,

$$
\mathrm{P}\left(\lim _{m \rightarrow \infty} \mathbf{q}_{\mathcal{O}_{m}}(t)=\mathbf{q}(t), \lim _{m \rightarrow \infty} \mathbf{x}_{\mathcal{O}_{m}}(t)=\mathbf{x}(t)\right)=1 .
$$

P5 For every finite time $t \geqslant 0$, constant $c \geqslant 0$, and non-negative functions $V, V_{m}: \mathcal{Q} \times \mathbb{R}^{n} \rightarrow$ $[0, \infty)$, such that $V_{m}(q, x)=V(q, x), \forall(q, x) \in \mathcal{O}_{m}, \forall m \in \mathbb{Z}_{\geqslant 1}$, we have that

$$
\left\{\begin{array} { l } 
{ V _ { m } ( \mathbf { q } _ { \mathcal { O } _ { m } } ( t ) , \mathbf { x } _ { \mathcal { O } _ { m } } ( t ) ) \text { is measurable } } \\
{ \mathrm { E } [ V _ { m } ( \mathbf { q } _ { \mathcal { O } _ { m } } ( t ) , \mathbf { x } _ { \mathcal { O } _ { m } } ( t ) ) ] \leqslant c , \forall m \in \mathbb { Z } _ { \geqslant 1 } }
\end{array} \Rightarrow \left\{\begin{array}{l}
V(\mathbf{q}(t), \mathbf{x}(t)) \text { is measurable } \\
\mathrm{E}[V(\mathbf{q}(t), \mathbf{x}(t))] \leqslant c .
\end{array}\right.\right.
$$

and also that

$$
\left\{\begin{array} { l } 
{ \int _ { 0 } ^ { t } V _ { m } ( \mathbf { q } _ { \mathcal { O } _ { m } } ( s ) , \mathbf { x } _ { \mathcal { O } _ { m } } ( s ) ) d s \text { is measurable } } \\
{ \mathrm { E } [ \int _ { 0 } ^ { t } V _ { m } ( \mathbf { q } _ { \mathcal { O } _ { m } } ( s ) , \mathbf { x } _ { \mathcal { O } _ { m } } ( s ) ) d s ] \leqslant c , \forall m \in \mathbb { Z } _ { \geqslant 1 } }
\end{array} \Rightarrow \left\{\begin{array}{l}
\int_{0}^{t} V(\mathbf{q}(s), \mathbf{x}(s)) d s \text { is measurable } \\
\mathrm{E}\left[\int_{0}^{t} V(\mathbf{q}(s), \mathbf{x}(s)) d s\right] \leqslant c .
\end{array}\right.\right.
$$

Proof of Lemma 1. Given an arbitrary finite time $t \geqslant 0$,

$$
\begin{aligned}
t<\mathbf{T}_{\max } \Rightarrow \sup _{s \leqslant t}\|\mathbf{x}(s)\|<\infty \Rightarrow \quad \exists m^{*}>0, \forall m \geqslant m^{*}: T_{\mathcal{O}_{m}}>t \quad \Rightarrow \\
\Rightarrow \lim _{m \rightarrow \infty} \mathbf{q}_{\mathcal{O}_{m}}(t)=\mathbf{q}(t), \lim _{m \rightarrow \infty} \mathbf{x}_{\mathcal{O}_{m}}(t)=\mathbf{x}(t) .
\end{aligned}
$$

Therefore

$$
\mathrm{P}\left(\lim _{m \rightarrow \infty} \mathbf{q}_{\mathcal{O}_{m}}(t)=\mathbf{q}(t), \lim _{m \rightarrow \infty} \mathbf{x}_{\mathcal{O}_{m}}(t)=\mathbf{x}(t)\right) \geqslant \mathrm{P}\left(\mathbf{T}_{\max }>t\right) \geqslant \mathrm{P}\left(\mathbf{T}_{\max }=\infty\right)=1,
$$

from which proposition $\mathrm{P} 4$ follows. To prove proposition P5, we first use a similar argument to prove that

$$
\mathrm{P}\left(\lim _{m \rightarrow \infty} V_{m}\left(\mathbf{q}_{\mathcal{O}_{m}}(t), \mathbf{x}_{\mathcal{O}_{m}}(t)\right)=V(\mathbf{q}(t), \mathbf{x}(t))\right)=1 .
$$

Since the $V_{m}$ are non-negative, we can then use Fatou's Lemma to conclude that $V(\mathbf{q}(t), \mathbf{x}(t))$ is measurable and

$$
\mathrm{E}[V(\mathbf{q}(t), \mathbf{x}(t))] \leqslant \liminf _{m \rightarrow \infty} \mathrm{E}\left[V_{m}\left(\mathbf{q}_{\mathcal{O}_{m}}(t), \mathbf{x}_{\mathcal{O}_{m}}(t)\right)\right] \leqslant c,
$$

from which (34) follows. A similar argument can be used to prove (35). 


\subsection{Lyapunov-based Analysis}

We say that a function $V: \mathcal{Q} \times \mathcal{X} \rightarrow \mathbb{R}$ is a candidate Lyapunov function for the SDSHS defined in Section 2.3 if it satisfies the following properties:

A12 For each fixed $q \in \mathcal{Q}$, the function $x \mapsto V(q, x)$ is continuously differentiable.

A13 $V$ is non-negative in the sense that $V(q, x) \geqslant 0, \forall q \in \mathcal{Q}, x \in \mathbb{R}^{n}$.

A14 $V$ is radially unbounded in $\mathbb{R}^{n}$ in the sense that, $\forall m>0$, the set

$$
\mathcal{O}_{m}:=\left\{(q, x) \in \mathcal{Q} \times \mathbb{R}^{n}: V(q, x)<m\right\}
$$

satisfies A11 (and also A10 because of A12).

The following result can be used to establish stability in probability and is adapted from [21, Theorem 2.1, p. 524], [19, Theorem 1, p. 38] and [19, Theorem 2, p. 39].

Theorem 4 (Stability of a SDSHS). Consider the SDSHS defined in Section 2.3 and an associated candidate Lyapunov function $V: \mathcal{Q} \times \mathbb{R}^{n} \rightarrow \mathbb{R}$.

P6 If

$$
L V(q, x) \leqslant \rho V, \quad \forall q \in \mathcal{Q}, x \in \mathbb{R}^{n}
$$

for some constant $\rho \geqslant 0$ then, for every initial condition $\mathbf{q}(0)=q_{0} \in \mathcal{Q}, \mathbf{x}(0)=x_{0} \in \mathbb{R}^{n}$, the solution to the SDSHS is globally defined with probability one, i.e., $\mathrm{P}\left(\mathbf{T}_{\max }=\infty\right)=1$.

P7 If (37) holds with $\rho=0$, i.e.,

$$
L V(q, x) \leqslant 0, \quad \forall q \in \mathcal{Q}, x \in \mathbb{R}^{n}
$$

then, for every $m>0$ and every initial condition $\mathbf{q}(0)=q_{0} \in \mathcal{Q}, \mathbf{x}(0)=x_{0} \in \mathbb{R}^{n}$,

$$
\mathrm{P}(\exists t \in[0, \infty): V(\mathbf{q}(t), \mathbf{x}(t)) \geqslant \mu) \stackrel{\text { wpo }}{\leqslant} \frac{V\left(q_{0}, x_{0}\right)}{\mu}, \quad \forall \mu \in(0, m] .
$$

P8 If, in addition to (38), $V$ is positive definite, in the sense that there exist functions $\gamma_{1}, \gamma_{2}$ of class $\mathcal{K}$ such that

$$
\gamma_{1}(\|x\|) \leqslant V(q, x) \leqslant \gamma_{2}(\|x\|), \quad \forall q \in \mathcal{Q}, x \in \mathbb{R}^{n}
$$

then the SDSHS is stable in probability (Definition D1).

P9 If, in addition to (38) and (40), $L V$ is negative definite, in the sense that there exists a function $\alpha$ of class $\mathcal{K}$ such that

$$
L V(q, x) \leqslant-\alpha(\|x\|), \quad \forall q \in \mathcal{Q}, x \in \mathbb{R}^{n}
$$

then the SDSHS is asymptotically stable in probability (Definition D2). 
In view of (25) or (26), the condition (38) essentially means that the expected value of $V(\mathbf{q}(t), \mathbf{x}(t))$ should decrease along solutions to the SDSHS. This non-increase on the average would hold $a$ fortiori if we demanded that

$$
\frac{\partial V(q, x)}{\partial x} f_{q}(x) \leqslant 0 \quad \text { and } \quad V\left(\phi_{\ell}(q, x)\right) \leqslant V(q, x), \quad \forall \ell \in \mathcal{L}, q \in \mathcal{Q}, x \in \mathbb{R}^{n},
$$

where the left-hand side inequality essentially requires $V(\mathbf{q}(t), \mathbf{x}(t))$ to decrease along the (deterministic) flows of $\dot{\mathbf{x}}=f_{q}(\mathbf{x})$ and the right-hand side inequality requires $V(\mathbf{q}(t), \mathbf{x}(t))$ not to increase at each reset time. However, (38) is weaker than (41) because, for (38) to hold the decrease only needs to occur in expected value, which permits some sample paths to exhibit strict increase on the value of $V(\mathbf{q}(t), \mathbf{x}(t))$ along flows of $\dot{\mathbf{x}}=f_{q}(\mathbf{x})$ and/or strict increase on the value of $V\left(\mathbf{q}(t), \mathbf{x}\left(\mathbf{t}_{k}\right)\right)$ at reset times.

The following results can be used to establish aggregate notions of stability. It assumes that the solution to the SDSHS is globally defined, which could be established, e.g., using proposition P6 in Theorem 4.

Theorem 5 (Mean stability of a SDSHS). Consider the SDSHS defined in Section 2.3, an associated candidate Lyapunov function $V: \mathcal{Q} \times \mathbb{R}^{n} \rightarrow \mathbb{R}$, and assume that the solution to the SDSHSis globally defined with probability one, i.e., $\mathrm{P}\left(\mathbf{T}_{\max }=\infty\right)=1$.

$\mathbf{P 1 0}$ If (38) holds, then the SDSHS is mean-V stable (Definition D3) and, for every initial condition $\mathbf{q}(0)=q_{0} \in \mathcal{Q}, \mathbf{x}(0)=x_{0} \in \mathbb{R}^{n}$,

$$
\mathrm{E}[V(\mathbf{q}(t), \mathbf{x}(t))] \leqslant V\left(q_{0}, x_{0}\right), \quad \forall t \geqslant 0 .
$$

and the solution to the SDSHS is globally defined with probability one, i.e., $\mathrm{P}\left(\mathbf{T}_{\max }=\infty\right)=1$.

$\mathbf{P 1 1}$ If

$$
L V(q, x) \leqslant-W(q, x), \quad \forall q \in \mathcal{Q}, x \in \mathbb{R}^{n},
$$

for a function $W: \mathcal{Q} \times \mathbb{R}^{n} \rightarrow \mathbb{R}$ such that $W(q, x) \geqslant 0, \forall q \in \mathcal{Q}, x \in \mathbb{R}^{n}$, then the $S D S H S$ is stochastic mean-W stable (Definition D4) and, for every initial condition $\mathbf{q}(0)=q_{0} \in \mathcal{Q}$, $\mathbf{x}(0)=x_{0} \in \mathbb{R}^{n}$,

$$
\int_{0}^{\infty} \mathrm{E}[W(\mathbf{q}(t), \mathbf{x}(t))] \leqslant V\left(q_{0}, x_{0}\right), \quad \forall t \geqslant 0
$$

$\mathbf{P 1 2}$ If

$$
L V(q, x) \leqslant-\rho V(q, x)+c, \quad \forall q \in \mathcal{Q}, x \in \mathbb{R}^{n},
$$

for constants $c \geqslant 0, \rho>0$, then the SDSHS is mean- $V$ stable (Definition D3) and, for every initial condition $\mathbf{q}(0)=q_{0} \in \mathcal{Q}, \mathbf{x}(0)=x_{0} \in \mathbb{R}^{n}$,

$$
\mathrm{E}[V(\mathbf{q}(t), \mathbf{x}(t))] \leqslant e^{-\rho t} V\left(q_{0}, x_{0}\right)+\frac{c}{\rho}\left(1-e^{-\rho t}\right), \quad \forall t \geqslant 0 .
$$

In case $c=0$, then the SDSHS is exponentially mean-V stable (Definition D6). 
P13 If

$$
L V(q, x) \leqslant-W(q, x)+c, \quad \forall q \in \mathcal{Q}, x \in \mathbb{R}^{n},
$$

for a constant $c \geqslant 0$ and a function $W: \mathcal{Q} \times \mathbb{R}^{n} \rightarrow \mathbb{R}$ such that $W(q, x) \geqslant 0, \forall q \in \mathcal{Q}, x \in \mathbb{R}^{n}$, then, for every initial condition $\mathbf{q}(0)=q_{0} \in \mathcal{Q}, \mathbf{x}(0)=x_{0} \in \mathbb{R}^{n}$,

$$
\lim _{T \rightarrow \infty} \frac{1}{T} \int_{0}^{T} \mathrm{E}[W(\mathbf{q}(t), \mathbf{x}(t))] d t \leqslant c
$$

Computationally effective systematic procedures to find Lyapunov functions for SDSHSs are generally not known, but a notable exception are the SDSHSs considered in Section 2.4 to emulate linear TTSHSs. We saw in Section 2.4 that the TTSIS defined by the vector field (15), the reset map (16), and a reset-time distribution $\mu$ for which there exists a probability density function $f$, i.e.,

$$
F(h):=\int_{[0, h]} \mu(d s)=\int_{0}^{h} f(s) d s, \quad \forall h \in[0, T), \quad T:=\sup \{h \in \mathbb{R}: F(h)<1\} \in \mathbb{R} \cup\{\infty\},
$$

can be emulated by a SDSHS with continuous state $(\mathbf{x}, \boldsymbol{\tau}) \in \mathbb{R}^{n} \times \mathbb{R}$; a vector field defined by

$$
\dot{\mathrm{x}}=A \mathbf{x}, \quad \dot{\tau}=1
$$

and a single reset map $\phi$ and transition intensity $\lambda$ defined by

$$
\phi(x, \tau):=(J x, 0), \quad \lambda(\tau):=\frac{f(\tau)}{1-F(\tau)}, \quad \forall x \in \mathbb{R}^{n}, \tau \in[0, T) .
$$

Theorem 6. Consider a TTSIS defined by the vector field (15), the reset map (16), and a reset-time distribution $\mu$, for which Assumption 1 holds. Further assume that the reset-time distribution $\mu$ can be defined by a probability density function, i.e., when there exists a function $f:[0, \infty) \rightarrow[0, \infty)$ such that (45) holds. This system is uniformly exponentially mean-square stable (Definition D6 with squared norm $W$ ) if and only if there exists a positive definite matrix-valued function $P$ : $[0, \infty) \rightarrow \mathbb{R}^{n \times n}$ and constants $c_{1}, c_{2}>0$ such that, for every positive definite matrices $S_{1}, S_{2} \in \mathbb{R}^{n}$,

$$
c_{1} I<P(\tau)<c_{2} I, \quad \dot{P}(\tau)=-S_{1}-A^{\prime} P(\tau)-P(\tau) A-\lambda(\tau)\left(J^{\prime} P(0) J-P(\tau)+S_{2}\right), \quad \forall \tau \in[0, T) .
$$

In this case, $V(x, \tau):=x^{\prime} P(\tau) x, \forall x \in \mathbb{R}^{n}, \tau \geqslant 0$ is a candidate Lyapunov function for which (43) holds with $c=0$ for the SDSHS defined by (46)-(47).

Example 2 (Estimation through a packet-switched network (cont.)). As mentioned before, the error dynamics of the state estimation error $\mathbf{e}$ for the networked control system considered in Example 2 can be modeled by the (single-mode) SDSHS in Figure 8, whose extended generator is of the form

$$
\begin{aligned}
L V(e):=\frac{\partial V(e)}{\partial e} A e+\lambda(e) & (V(0)-V(e)) \\
+ & \frac{1}{2 \epsilon}(V(e+\sqrt{\epsilon} b)-V(e))+\frac{1}{2 \epsilon}(V(e-\sqrt{\epsilon} b)-V(e)), \quad \forall e \in \mathbb{R}^{n},
\end{aligned}
$$


for a given function $V: \mathbb{R}^{n} \rightarrow \mathbb{R}$. The first step in analyzing the resulting SDSHS is to establish global existence of solution. To this effect we consider a candidate Lyapunov function of the form

$$
V_{\text {global }}(e):=\|e\|^{2}+c, \quad \forall e \in \mathbb{R}^{n},
$$

for some appropriately chosen constant $c \geqslant 0$. Applying the extended generator (48) to $V_{\text {global }}$, leads to

$$
\begin{aligned}
L V_{\text {global }}(e)=e^{\prime}\left(A+A^{\prime}-\lambda(e)\right) e+b^{\prime} b & \leqslant \sigma_{\max }\left[A+A^{\prime}\right]\|e\|^{2}+b^{\prime} b \\
& =\sigma_{\max }\left[A+A^{\prime}\right]\left(V_{\text {global }}(e)-c\right)+b^{\prime} b \quad \forall e \in \mathbb{R}^{n},
\end{aligned}
$$

where $\sigma_{\max }\left[A+A^{\prime}\right]$ denotes the largest singular value of $A+A^{\prime}$. Picking $c:=b^{\prime} b / \sigma_{\max }\left[A+A^{\prime}\right]$, we conclude that $L V_{\text {global }}(e) \leqslant \sigma_{\max }\left[A+A^{\prime}\right] V_{\text {global }}(e), \forall e \in \mathbb{R}^{n}$ and we can use proposition $\mathrm{P} 6$ in Theorem 4 to conclude global existence of solution with probability one, regardless of our choice for the intensity $\lambda(e)$.

To study mean stability of the SDSHS, we consider polynomial candidate Lyapunov functions of the form

$$
V_{\alpha}(e):=\left(e^{\prime} P e\right)^{\alpha}, \quad \forall e \in \mathbb{R}^{n},
$$

with $\alpha \geqslant 1$. Applying now the extended generator (48) to $V_{\alpha}$, leads to

$$
\begin{aligned}
& L V_{\alpha}(e)=\alpha\left(e^{\prime} P e\right)^{\alpha-1} e^{\prime}\left(P A+A^{\prime} P\right) e-\lambda(e)\left(e^{\prime} P e\right)^{\alpha} \\
& +\frac{\left((e+\sqrt{\epsilon} b)^{\prime} P(e+\sqrt{\epsilon} b)\right)^{\alpha}+\left((e-\sqrt{\epsilon} b)^{\prime} P(e-\sqrt{\epsilon} b)\right)^{\alpha}-2\left(e^{\prime} P e\right)^{\alpha}}{2 \epsilon}, \quad \forall e \in \mathbb{R}^{n}
\end{aligned}
$$

which can be written as

$$
L V_{\alpha}(e)=-\rho V(e)+c(e), \quad \forall e \in \mathbb{R}^{n}
$$

for any constant $\rho>0$, provided that we define

$$
\begin{aligned}
c(e):=\alpha\left(e^{\prime} P e\right)^{\alpha-1} e^{\prime}(P A+ & \left.A^{\prime} P-\frac{\lambda(e)-\rho}{\alpha} P\right) e \\
& +\frac{\left((e+\sqrt{\epsilon} b)^{\prime} P(e+\sqrt{\epsilon} b)\right)^{\alpha}+\left((e-\sqrt{\epsilon} b)^{\prime} P(e-\sqrt{\epsilon} b)\right)^{\alpha}-2\left(e^{\prime} P e\right)^{\alpha}}{2 \epsilon} .
\end{aligned}
$$

To check whether or not $c(e)$ is bounded, we can use the fact that, since $P$ is positive definite, the dominante terms in $c(e)$ as $e \rightarrow \infty$ are the powers in $\left(e^{\prime} P e\right)^{\alpha}$ :

$$
\alpha\left(e^{\prime} P e\right)^{\alpha-1} e^{\prime}\left(P A+A^{\prime} P-\frac{\lambda(e)-\rho}{\alpha}\right) e
$$

which is negative as long as

$$
P A+A^{\prime} P-\frac{\lambda(e)-\rho}{\alpha} P<0 .
$$

In case $\lambda(e)$ is radially unbounded, this is always the case for sufficiently large $e$ and we conclude that

$$
L V_{\alpha}(e) \leqslant-\rho V(e)+c_{\max }, \quad \forall e \in \mathbb{R}^{n}, \quad \quad c_{\max }=\sup _{e \in \mathbb{R}^{n}} c(e)<\infty
$$


Using proposition P11 in Theorem 5 we conclude that if the intensity $\lambda(e)$ is radially unbounded, the SDSHS is mean- $V_{\alpha}$ stable and consequently the $2 \alpha$-moment of the error $\mathrm{E}\left[\|e\|^{2 \alpha}\right]$ is bounded, for every $\alpha \geqslant 1$.

In case $\lambda(e)$ eventually "saturates" for large $e$, i.e.,

$$
\exists \kappa>0:\|e\|>\kappa \Rightarrow \lambda(e)=\lambda_{\infty}, \quad \forall e \in \mathbb{R}^{n},
$$

then mean- $V_{\alpha}$ stability can be established if we can find a positive definite matrix $P$ and a positive constant $\rho$ for which

$$
P A+A^{\prime} P-\frac{\kappa-\rho}{\alpha} P=P\left(A-\frac{\kappa-\rho}{2 \alpha} I\right)+\left(A-\frac{\kappa-\rho}{2 \alpha} I\right)^{\prime} P<0 .
$$

This turns out to be the case if and only if all the eigenvalues of $A$ have real part strictly less than $\frac{\kappa}{2 \alpha}$, i.e.,

$$
\lambda_{\Re}(A)<\frac{\kappa}{2 \alpha},
$$

where $\lambda_{\Re}(A)$ denotes the real part of the eigenvalue of $A$ with largest real part. In case $A$ has no eigenvalues with positive real part, this condition always holds and we again can conclude that the $2 \alpha$-moment of the error $\mathrm{E}\left[\|e\|^{2 \alpha}\right]$ is bounded, for every $\alpha \geqslant 1$. However, if $A$ has any eigenvalue with positive real part, the condition above is equivalent to

$$
\alpha<\frac{\kappa}{2 \lambda_{\Re}(A)},
$$

which means that only some of the moments $\mathrm{E}\left[\|e\|^{2 \alpha}\right]$ may be bounded.

\subsection{Martingales and Doob's inequality}

While complete proofs of the results summarized in Section 5.3 is beyond the scope of this paper, it is worth describing the key steps behind the Lyapunov-based analysis of SDSHSs, as these may enable the reader to construct alternative results. These proofs rely heavily on the concept of Martingale and the pioneering results by Doob.

We recall that a stochastic process $\mathbf{M}(t), t \geqslant 0$ is called a supermartingale for a filtration $\left\{\mathcal{F}_{t}: t \geqslant 0\right\}$ if

$$
\mathrm{E}[\mathbf{M}(t)]<\infty, \quad \forall t \geqslant 0, \quad \mathrm{E}\left[\mathbf{M}(t) \mid \mathcal{F}_{\tau}\right] \stackrel{\text { wpo }}{\leqslant} \mathbf{M}(\tau), \quad \forall t \geqslant \tau \geqslant 0 .
$$

Intuitively, this means that the current value of $\mathbf{M}(\tau)$ provides an upper bound on future expectations of $\mathbf{M}(t), t \geqslant \tau$. Because a supermartingale only decreases "on average," specific sample paths may occasionally increase. However, the probability of a large increase can be upper bounded through Doob's martingale inequality [19, Section I-7.]:

Lemma 2 (Doob's martingale inequality). If $\mathbf{M}(t), t \geqslant 0$ is a non-negative supermartingale for the filtration $\left\{\mathcal{F}_{t}: t \geqslant 0\right\}$, then

$$
\mathrm{P}(\exists t \in[0, \infty): \mathbf{M}(t) \geqslant \mu) \stackrel{\text { wpo } \mathrm{E}[\mathbf{M}(0)]}{\mu}, \quad \forall \mu>0 .
$$




\subsubsection{Proof of Theorem 4}

In Lyapunov-based stability proofs, the supermartingales of interest are of the form

$$
\mathbf{M}_{\mathcal{O}_{m}}(t):=e^{-\rho t} V\left(\mathbf{q}_{\mathcal{O}_{m}}(t), \mathbf{x}_{\mathcal{O}_{m}}(t)\right), \quad \forall t \geqslant 0
$$

with $\rho \geqslant 0$ and sets $\mathcal{O}_{m}$ of the form (36). To verify that this process is a supermartingale under (37) in $\mathrm{P} 6$, we use (32) to conclude that

$$
\begin{aligned}
\frac{d \mathrm{E}\left[\mathbf{M}_{\mathcal{O}_{m}}(t) \mid \mathcal{F}_{\tau}\right]}{d t} & =e^{-\rho t}\left[\frac{d \mathrm{E}\left[V\left(\mathbf{q}_{\mathcal{O}_{m}}(t), \mathbf{x}_{\mathcal{O}_{m}}(t)\right) \mid \mathcal{F}_{\tau}\right]}{d t}-\rho \mathrm{E}\left[V\left(\mathbf{q}_{\mathcal{O}_{m}}(t), \mathbf{x}_{\mathcal{O}_{m}}(t)\right) \mid \mathcal{F}_{\tau}\right]\right] \\
& =e^{-\rho t} \mathrm{E}\left[L_{\mathcal{O}_{m}} V\left(\mathbf{q}_{\mathcal{O}_{m}}(t), \mathbf{x}_{\mathcal{O}_{m}}(t)\right)-\rho V\left(\mathbf{q}_{\mathcal{O}_{m}}(t), \mathbf{x}_{\mathcal{O}_{m}}(t)\right) \mid \mathcal{F}_{\tau}\right], \quad \forall t \stackrel{\text { ae }}{\in}[\tau, 0), \forall \tau \geqslant 0 .
\end{aligned}
$$

where $\left\{\mathcal{F}_{t}: t \geqslant 0\right\}$ is the natural filtration of the Markov process $\mathbf{z}_{\mathcal{O}_{m}}:=\left(\mathbf{q}_{\mathcal{O}_{m}}, \mathbf{x}_{\mathcal{O}_{m}}\right)$. On the other hand, from (33) and (37), we conclude that

$$
L_{\mathcal{O}_{m}} V(q, x) \leqslant \rho V(q, x), \quad \forall q \in \mathcal{Q}, x \in \mathbb{R}^{n}
$$

and therefore

$$
\frac{d \mathrm{E}\left[\mathbf{M}_{\mathcal{O}_{m}}(t) \mid \mathcal{F}_{\tau}\right]}{d t} \leqslant 0, \quad \forall t \stackrel{\text { ae }}{\in}[\tau, \infty), \forall \tau \geqslant 0
$$

which leads to

$$
\mathrm{E}\left[\mathbf{M}_{\mathcal{O}_{m}}(t) \mid \mathcal{F}_{\tau}\right] \stackrel{\text { wpo }}{\leqslant} \mathbf{M}_{\mathcal{O}_{m}}(\tau), \quad \forall t \stackrel{\text { ae }}{\in}[\tau, \infty), \forall \tau \geqslant 0
$$

and, in particular for $\tau=0$,

$$
\mathrm{E}\left[\mathbf{M}_{\mathcal{O}_{m}}(t)\right] \stackrel{\text { wpo }}{\leqslant} \mathbf{M}_{\mathcal{O}_{m}}(0)=V\left(q_{0}, x_{0}\right), \quad \forall t \geqslant 0,
$$

confirming that (49) is indeed a non-negative supermartingale. It then follows from Doob's martingale inequality that

$$
\mathrm{P}\left(\exists t \in[0, \infty): e^{-\rho t} V\left(\mathbf{q}_{\mathcal{O}_{m}}(t), \mathbf{x}_{\mathcal{O}_{m}}(t)\right) \geqslant \mu\right) \stackrel{\text { wpo }}{\leqslant} \frac{V\left(q_{0}, x_{0}\right)}{\mu}, \quad \forall \mu>0 .
$$

To establish global existence of solution, we use the fact that we cannot have $\mathbf{T}_{\max }<\infty$ if $\mathbf{x}(t)$ remains in a bounded set. From this and the fact that the Lyapunov function is radially unbounded (per A14), we conclude that for every sample path for which $\mathbf{T}_{\max }<\infty$, the Lyapunov function must take arbitrarily large values in the (bounded) interval $\left[0, \mathbf{T}_{\max }\right)$, i.e.,

$$
\mathbf{T}_{\max }<\infty \Rightarrow \forall m>0, \exists t \in\left[0, \mathbf{T}_{\max }\right), V\left(\mathbf{q}_{\mathcal{O}_{m}}(t), \mathbf{x}_{\mathcal{O}_{m}}(t)\right) \geqslant e^{\rho \mathbf{T}_{\max }} m
$$

and therefore

$$
\begin{aligned}
\mathrm{P}\left(\mathbf{T}_{\max }<\infty\right) & \leqslant \mathrm{P}\left(\forall m>0, \exists t \in\left[0, \mathbf{T}_{\max }\right), V\left(\mathbf{q}_{\mathcal{O}_{m}}(t), \mathbf{x}_{\mathcal{O}_{m}}(t)\right) \geqslant e^{\rho \mathbf{T}_{\max }} m\right) \\
& \leqslant \mathrm{P}\left(\forall m>0, \exists t \in\left[0, \mathbf{T}_{\max }\right), V\left(\mathbf{q}_{\mathcal{O}_{m}}(t), \mathbf{x}_{\mathcal{O}_{m}}(t)\right) \geqslant e^{\rho t} m\right)
\end{aligned}
$$




$$
\begin{aligned}
& \leqslant \mathrm{P}\left(\forall m>0, \exists t \in[0, \infty), V\left(\mathbf{q}_{\mathcal{O}_{m}}(t), \mathbf{x}_{\mathcal{O}_{m}}(t)\right) \geqslant e^{\rho t} m\right) \\
& =\mathrm{P}\left(\bigcap_{m>0}\left\{\omega \in \Omega: \exists t \in[0, \infty), V\left(\mathbf{q}_{\mathcal{O}_{m}}(t), \mathbf{x}_{\mathcal{O}_{m}}(t)\right) \geqslant e^{\rho t} m\right\}\right) \\
& =\lim _{m \rightarrow \infty} \mathrm{P}\left(\exists t \in[0, \infty), V\left(\mathbf{q}_{\mathcal{O}_{m}}(t), \mathbf{x}_{\mathcal{O}_{m}}(t)\right) \geqslant e^{\rho t} m\right),
\end{aligned}
$$

where we used the fact that for any infinite sequence of measurable sets $E_{1} \supset E_{2} \supset E_{3} \supset \cdots$, we have that $\mathrm{P}\left(\cap_{n \geqslant 1} E_{n}\right)=\lim _{n \rightarrow \infty} \mathrm{P}\left(E_{n}\right)$. If we now use (51) with $\mu=m$, we conclude that

$$
\mathrm{P}\left(\mathbf{T}_{\max }<\infty\right) \leqslant \lim _{m \rightarrow \infty} \frac{V\left(q_{0}, x_{0}\right)}{m}=0, \quad \forall T \in[0, \infty),
$$

which means that the solution to the SDSHS must be globally defined with probability one, completing the proof of $\mathrm{P} 6$.

The proof of $\mathrm{P} 7$ follows from (51) with $\rho=0$, since the definitions of the set $\mathcal{O}_{m}$ and the stopped processes allow us to conclude that, for every $\mu \leqslant m$,

$$
\exists t \in[0, \infty): V(\mathbf{q}(t), \mathbf{x}(t)) \geqslant \mu \quad \Rightarrow \quad \exists t \in[0, \infty): V\left(\mathbf{q}_{\mathcal{O}_{m}}(t), \mathbf{x}_{\mathcal{O}_{m}}(t)\right) \geqslant \mu
$$

and therefore, we can use (51) with $\rho=0$ to conclude that

$$
\mathrm{P}(\exists t \in[0, \infty): V(\mathbf{q}(t), \mathbf{x}(t)) \geqslant \mu) \leqslant \mathrm{P}\left(\exists t \in[0, \infty): V\left(\mathbf{q}_{\mathcal{O}_{m}}(t), \mathbf{x}_{\mathcal{O}_{m}}(t)\right) \geqslant \mu\right) \stackrel{\text { wpo }}{\leqslant} \frac{V\left(q_{0}, x_{0}\right)}{\mu},
$$

which establishes P7.

The proposition P8 is a direct consequence of (39), which can be used to provide an explicit estimate on the state bound $\delta$, as a function of $\rho, \epsilon$ in the definition in (14): for given $\epsilon, \rho>0$, we simply need to pick $\mu, \delta>0$ sufficiently small so that

$$
\|\mathbf{x}(t)\|>\rho \quad \Rightarrow \quad \text { and } \quad\left\|x_{0}\right\| \leqslant \delta \quad \Rightarrow \quad \frac{V\left(q_{0}, x_{0}\right)}{\mu} \leqslant \epsilon .
$$

The existence of such $\mu, \delta>0$ is guaranteed by (40), as we can pick $\mu:=\gamma_{1}(\rho)$ and $\delta<\gamma_{2}^{-1}(\epsilon \mu)$. In this case, for every initial condition $\mathbf{q}(0)=q_{0} \in \mathcal{Q}, \mathbf{x}(0)=x_{0} \in \mathbb{R}^{n}$ with $\left\|x_{0}\right\| \leqslant \delta$, we have that (14) holds.

We refer the reader to [19, Theorem 2,p. 39] for the proof of P9, which is somewhat more technical.

\subsubsection{Proof of Theorem 5}

The bounds on the expected values found in Theorem 5 are obtained by first using Dynkin's inequality for stopped process associated with sets $\mathcal{O}_{m}$ of the form (36) and then making $m \rightarrow \infty$. Specifically, to establish P10 in Theorem 5, we use (50) with $\rho=0$ to conclude that

$$
\mathrm{E}\left[V\left(\mathbf{q}_{\mathcal{O}_{m}}(t), \mathbf{x}_{\mathcal{O}_{m}}(t)\right)\right] \leqslant V\left(q_{0}, x_{0}\right), \quad \forall t \geqslant 0 .
$$

The result then follows from proposition P5 in Lemma 1 [with $V_{m}:=V, \forall m$ and $c:=V\left(q_{0}, x_{0}\right)$ ]. 
To prove proposition P11 in Theorem 5, first note that

$$
L V(q, x) \leqslant-W(q, x) \quad \Rightarrow \quad L_{\mathcal{O}_{m}} V(q, x) \leqslant-W_{\mathcal{O}_{m}}(q, x):= \begin{cases}-W(q, x) & (q, x) \in \mathcal{O}_{m} \\ 0 & (q, x) \notin \mathcal{O}_{m}\end{cases}
$$

We therefore conclude from (31) in Theorem 3 that, $\forall t \geqslant \tau:=0$,

$$
\begin{aligned}
\mathrm{E}\left[V\left(\mathbf{q}_{\mathcal{O}_{m}}(t), \mathbf{x}_{\mathcal{O}_{m}}(t)\right)\right] & =V\left(q_{0}, x_{0}\right)+\int_{0}^{t} \mathrm{E}\left[L_{\mathcal{O}_{m}} V\left(\mathbf{q}_{\mathcal{O}_{m}}(s), \mathbf{x}_{\mathcal{O}_{m}}(s)\right)\right] d s \\
& \leqslant V\left(q_{0}, x_{0}\right)-\int_{0}^{t} \mathrm{E}\left[W_{\mathcal{O}_{m}}\left(\mathbf{q}_{\mathcal{O}_{m}}(s), \mathbf{x}_{\mathcal{O}_{m}}(s)\right)\right] d s
\end{aligned}
$$

and, consequently,

$$
\int_{0}^{t} \mathrm{E}\left[W_{\mathcal{O}_{m}}\left(\mathbf{q}_{\mathcal{O}_{m}}(s), \mathbf{x}_{\mathcal{O}_{m}}(s)\right)\right] d s \leqslant V\left(q_{0}, x_{0}\right), \quad \forall t \geqslant 0 .
$$

We now use proposition P5 in Lemma 1 [with $V_{m}:=W_{\mathcal{O}_{m}}$ and $c:=V\left(q_{0}, x_{0}\right)$ ] to conclude that

$$
\int_{0}^{t} \mathrm{E}[W(\mathbf{q}(s), \mathbf{x}(s))] d s \leqslant V\left(q_{0}, x_{0}\right), \quad \forall t \geqslant 0
$$

from which (42) follows.

To prove proposition P12 of Theorem 5, we can use an auxiliary SDSHS obtained by augmenting the continuous state of our original SDSHS with a time variable $\mathbf{T}$ that is initialize at $\mathbf{T}(0)=0$, that flows according to $\dot{\mathbf{T}}=1$ and that is not affected by any of the resets, which means that $\mathbf{T}(t) \stackrel{\text { wpo }}{=} t, \forall t$. The extended generator $\bar{L}$ of this auxiliary system can be constructed from the extended generator $L$ of the original system using

$$
\bar{L} \bar{V}(q, x, T):=L \bar{V}(q, x, T)+\frac{\partial \bar{V}(q, x, T)}{\partial T}, \quad \forall q \in \mathcal{Q}, x \in \mathbb{R}^{n}, T \geqslant 0,
$$

where in applying the extended generator $L$ of the original system to $\bar{V}(q, x, T)$, we regard $T$ as a constant parameter. If we now use the original candidate Lyapunov function $V(q, x)$ to construct a candidate Lyapunov function $\bar{V}(q, x, T)$ for the auxiliary SDSHS as follows

$$
\bar{V}(q, x, T):=e^{\rho T} V(q, x), \quad \forall q \in \mathcal{Q}, x \in \mathbb{R}^{n}, T \in[0, \infty),
$$

we conclude from (43) that

$$
\bar{L} \bar{V}(q, x, T):=e^{\rho T}(L V(q, x)+\rho V(q, x)) \leqslant e^{\rho T} c, \quad \forall q \in \mathcal{Q}, x \in \mathbb{R}^{n}, T \in[0, \infty) .
$$

Using (31) for the stopped processes of the auxiliar system associated with sets $\mathcal{O}_{m}$ of the form (36) defined using the new Lyapunov function $\bar{V}(q, x, T)$, we conclude that

$$
\mathrm{E}\left[\bar{V}\left(\mathbf{q}_{\mathcal{O}_{m}}(t), \mathbf{x}(t)_{\mathcal{O}_{m}}, \mathbf{T}(t)\right)\right] \leqslant \bar{V}\left(q_{0}, x_{0}, 0\right)+\int_{0}^{t} e^{\rho s} c d s=\bar{V}\left(q_{0}, x_{0}, 0\right)+\frac{c}{\rho}\left(e^{\rho t}-1\right), \quad \forall t \geqslant 0 .
$$

We can then use proposition P5 in Lemma 1 [with $V_{m}:=\bar{V}, \forall m$ and $c:=\bar{V}\left(q_{0}, x_{0}, 0\right)+c / \rho\left(e^{\rho t}-1\right)$ ] to go back to the non-stopped processes and conclude that

$$
\mathrm{E}[\bar{V}(\mathbf{q}(t), \mathbf{x}(t), \mathbf{T}(t))] \leqslant \bar{V}\left(q_{0}, x_{0}, 0\right)+\frac{c}{\rho}\left(e^{\rho t}-1\right) \quad \forall t \geqslant 0
$$

from which (44) follows.

We refer the reader to [19, Theorem 6, p. 50] for the proof of $\mathrm{P} 13$, which is a variation of the proofs discussed above. 


\section{Conclusions and Open Research Directions}

Stochastic hybrid systems provide an attractive tool to model and analyze networked control systems in which network-induced events (such as the departure and arrival of network packets) introduce stochastic instantaneous changes in the systems' states. We presented several tools for the analysis of such systems and illustrated their use in the context of a few simple examples.

While significant progress has been made towards the analysis of SHSs, several important problems remain open: The stability results for linear TTSHS are fairly complete, but the study of TTSHS with nonlinear vector fields or reset maps remains mostly open. The analysis of SDSHS also presents several open problems; most notably the development of systematic methods for the construction of Lyapunov functions, perhaps based on stochastic counter parts of backstepping or similar procedures. A notable omission in this paper is an explicit discussion on recurrence, i.e., the property that the state of a stochastic system returns infinitely often to a particular subset of the state space with high probability. This notion has proved to be useful in numerous contexts, including the introduction of stochastic notions of input-to-state stability (ISS) [23, 24].

\section{Acknowledgements}

The author thanks Farshad Pour Safaei and Duarte Antunes for multiple suggestions that greatly improved the presentation of this paper.

\section{References}

[1] D. Antunes, J. P. Hespanha, and C. Silvestre. Impulsive systems triggered by superposed renewal processes. In Proc. of the 49th Conf. on Decision and Contr., Dec. 2010. (cited in p. 20)

[2] D. Antunes, J. P. Hespanha, and C. Silvestre. Volterra integral approach to impulsive renewal systems: Application to networked control. IEEE Trans. on Automat. Contr., 57:607-619, Mar. 2012. (cited in p. 7, 10, 19, 20)

[3] D. Antunes, J. P. Hespanha, and C. Silvestre. Stability of networked control systems with asynchronous renewal links: An impulsive systems approach. Automatica, 49(2):402-413, Feb. 2013. (cited in p. 3, 7)

[4] D. Antunes, J. P. Hespanha, and C. Silvestre. Stochastic hybrid systems with renewal transitions: Moment analysis with applications to networked control systems with delays. SIAM J. Contr. Optimization, 51(2):1481-1499, Feb. 2013. (cited in p. 20)

[5] K. Åström and B. Bernhardsson. Comparison of Riemann and Lebesgue sampling for first order stochastic systems. In Proc. of the 41st Conf. on Decision and Contr., volume 2, pages 2011-2016, Dec. 2002. (cited in p. 13)

[6] M. L. Bujorianu and J. Lygeros. Towards a general theory of stochastic hybrid systems. In H. A. P. Blom and J. Lygeros, editors, Stochastic Hybrid Systems, volume 337 of Lect. Notes in Contr. and Inform. Sci., pages 3-30. Springer-Verlag, Berlin, 2006. (cited in p. 4) 
[7] C. G. Cassandras and J. Lygeros, editors. Stochastic Hybrid Systems: Recent Developments and Research Trends. Number 24 in Control Engineering Series. CRC Press, Boca Raton, Nov. 2006. (cited in p. 4)

[8] A. Cervin and B. Lincoln. Jitterbug 1.23 Reference Manual. Dept. of Automatic Control, Lund University, Sweden, July 2010. (cited in p. 4)

[9] R. Cogill, S. Lall, and J. P. Hespanha. A constant factor approximation algorithm for eventbased sampling. In J. C. Willems, S. Hara, Y. Ohta, and H. Fujioka, editors, Perspectives in Mathematical System Theory, Control, and Signal Processing, number 398 in Lecture Notes in Control and Information Sciences, chapter A Constant Factor Approximation Algorithm for Event-Based Sampling, pages 51-60. Springer-Verlag, Berlin, Mar. 2010. (cited in p. 13)

[10] O. L. V. Costa, M. D. Fragoso, and M. G. Todorov. Continuous-Time Markov Jump Linear Systems. Probability and Its Applications. Springer-Verlag, Berlin, 2013. (cited in p. 4)

[11] M. H. A. Davis. Markov models and optimization. Monographs on statistics and applied probability. Chapman \& Hall, London, UK, 1993. (cited in p. 4, 22, 24)

[12] G. B. Folland. Real Analysis: Modern Techniques and Their Applications. Pure and Applied Mathematics. John Wiley \& Sons, New York, 1984. (cited in p. 21)

[13] M. K. Ghosh, A. Arapostathis, and S. Marcus. Ergodic control of switching diffusions. SIAM J. Contr. Optimization, 35(6):1952-1988, Nov. 1997. (cited in p. 4)

[14] J. P. Hespanha. Polynomial stochastic hybrid systems. In M. Morari and L. Thiele, editors, Hybrid Systems: Computation and Control, number 3414 in Lect. Notes in Comput. Science, pages 322-338. Springer-Verlag, Berlin, Mar. 2005. (cited in p. 4)

[15] J. P. Hespanha. Modeling and analysis of stochastic hybrid systems. IEE Proc. - Control Theory 83 Applications, Special Issue on Hybrid Systems, 153(5):520-535, 2007. (cited in p. 4)

[16] J. P. Hespanha, P. Naghshtabrizi, and Y. Xu. A survey of recent results in networked control systems. Proc. of IEEE Special Issue on Technology of Networked Control Systems, 95(1): 138-162, Jan. 2007. (cited in p. 1)

[17] J. Hu, J. Lygeros, and S. Sastry. Towards a theory of stochastic hybrid systems. In N. A. Lynch and B. H. Krogh, editors, Hybrid Systems: Computation and Control, volume 1790 of Lect. Notes in Comput. Science, pages 160-173. Springer, 2000. (cited in p. 4)

[18] O. Imer and T. Basar. Optimal estimation with limited measurements. Int. J. of Systems, Control and Comm., 2(1):5-29, 2010. (cited in p. 13)

[19] H. J. Kushner. Stochastic Stability and Control, volume 33 of Mathematics in Science and Engineering. Academic Press, New York, 1967. (cited in p. 23, 26, 30, 32, 33)

[20] M. Mariton. Jump Linear Systems in Automatic Control. CRC Press, Feb. 1990. (cited in p. 4)

[21] S. P. Meyn and R. L. Tweedie. Stability of Markovian processes iii: Foster-Lyapunov criteria for continuous-time processes. Advances in Applied Probability, 25(3):518-548, 19931993. (cited in p. 26) 
[22] A. R. Teel. Lyapunov conditions certifying stability and recurrence for a class of stochastic hybrid systems. Annual Reviews in Control, 37(1):1-24, Apr. 2013. (cited in p. 4,6)

[23] A. R. Teel, A. Subbaraman, and A. Sferlazza. Stability analysis for stochastic hybrid systems: a survey. Submitted to publication. (cited in p. 3, 4, 34)

[24] A. R. Teel, J. P. Hespanha, and A. Subbaraman. Equivalent characterizations of input-to-state stability for stochastic discrete-time systems. IEEE Trans. on Automat. Contr., 59(2), Feb. 2014. (cited in p. 34)

[25] Y. Tipsuwan and M.-Y. Chow. Control methodologies in networked control systems. Control Engineering Practice, Special Section on Control Methods for Telecommunication, 11(10): 1099-1111, 2003. (cited in p. 1)

[26] Y. Xu and J. P. Hespanha. Communication logic design and analysis for networked control systems. In L. Menini, L. Zaccarian, and C. T. Abdallah, editors, Current trends in nonlinear systems and control. Birkhäuser, Boston, 2006. (cited in p. 13)

[27] G. Yin and C. Zhu. Hybrid Switching Diffusions: Properties and Applications. Number 63 in Stochastic Modelling and Applied Probability. Springer, New York, 2000. (cited in p. 4)

[28] J. K. Yook, D. M. Tilbury, and N. R. Soparkar. Trading computation for bandwidth: Reducing communication in distributed control systems using state estimators. IEEE Trans. on Contr. Syst. Tech., 10(4):503-518, July 2002. (cited in p. 13)

[29] W. Zhang, M. S. Branicky, and S. M. Phillips. Stability of networked control systems. IEEE Contr. Syst. Mag., pages 84-99, Feb. 2001. (cited in p. 1)

\section{Biographic Information}

João P. Hespanha received his the Ph.D. degree in electrical engineering and applied science from Yale University, New Haven, Connecticut in 1998. From 1999 to 2001, he was Assistant Professor at the University of Southern California, Los Angeles. He moved to the University of California, Santa Barbara in 2002, where he currently holds a Professor position with the Department of Electrical and Computer Engineering. Prof. Hespanha is the Chair of the Department of Electrical and Computer Engineering and a member of the Executive Committee for the Institute for Collaborative Biotechnologies (ICB).

His current research interests include hybrid and switched systems; multi-agent control systems; distributed control over communication networks (also known as networked control systems); the use of vision in feedback control; stochastic modeling in biology; and network security.

Prof. Hespanha is the recipient of the Yale University's Henry Prentiss Becton Graduate Prize for exceptional achievement in research in Engineering and Applied Science, a National Science Foundation CAREER Award, the 2005 best paper award at the 2nd Int. Conf. on Intelligent Sensing and Information Processing, the 2005 Automatica Theory/Methodology best paper prize, the 2006 George S. Axelby Outstanding Paper Award, and the 2009 Ruberti Young Researcher Prize. Prof. Hespanha is a Fellow of the IEEE and an IEEE distinguished lecturer since 2007. 